\title{
Systemic Risk and Centralized Clearing of OTC derivatives: A Network Approach
}

Svetlana Borovkova (VU Amsterdam)

\author{
AND
}

Hicham Lalaoui El Mouttalibi (PwC Nederland)

December 11, 2013 


\begin{abstract}
In September 2009, G20 paved the way for the mandatory central clearing of over-the-counter (OTC) derivatives, which came into effect in December 2012. This new regulation involves a central clearing counterparty $(C C P)$ : a financial institution acting as an intermediary between buyers and sellers of OTC derivatives. The rationale behind this regulation is that, by removing bilateral agreements, CCPs will absorb the risks facing individual firms and act as a cushion in the event of market stress. However, this increases the systemic importance of CCPs within the financial system.

In this paper, we analyze the effect of central clearing of OTC derivatives on the financial system stability by means of network simulation approach. We build simple but realistic networks of financial firms, connected by bilateral links and via a single CCP. We simulate balance sheets of firms and introduce shocks to the system to simulate defaults. The default mechanism and shock absorption in presence of the CCP is modeled in the way that maximally reflects the reality. We run Monte Carlo simulations of the networks' evolution and obtain their default and contagion characteristics. We analyze the likelihood of the CCP's default and compare the stability of the financial network with and without the CCP for various network configurations and market scenarios.

We find that, for a homogeneous financial system, the presence of the CCP increases the network's stability and the probability of the CCP's failure is virtually zero. However, for nonhomogeneous financial networks, we find the opposite effects: the presence of the CCP leads in this case to a disproportionately large probability of contagion defaults, especially for smaller financial firms. Furthermore, we find that the probability of the CCP failure is substantial in this case, regardless of the capitalization requirements. In all, we find that non-homogeneous networks exhibit greater instability and contagion in the presence of the CCP: a worrying fact, given that any real financial system is highly inhomogeneous in terms of size and concentration.
\end{abstract}

Keywords: central clearing, random networks, core-periphery structure, OTC derivatives, contagion. 


\section{Summary}

The 2008 financial crisis has led policymakers to implement legislation aimed at reducing the likelihood of future crisis and mitigating counterparty credit risk in the OTC derivatives market. The Dodd-Frank act and European Market Infrastructure Regulations (EMIR) mandate the central clearing of OTC derivatives transactions through central clearing counterparties (CCPs). The activities of CCPs have been of great benefit in various financial markets, e.g., exchange-traded futures and options markets, where their main purpose was to shield their clearing members from counterparty credit risk. The expansion of CCPs to OTC derivatives markets is meant to achieve the same effect. However, the envisioned CCPs will be crucial players in the financial system, hence a failure of a CCP can lead to catastrophic social losses. Further, clearing and margining mechanisms envisioned by CCPs are of great concern to many financial institutions who believe that the costs of central clearing (in terms of e.g., liquidity) will be prohibitive, thus inducing these institutions to pull out of OTC derivatives markets altogether.

This paper studies how contagion and system risk is impacted by CCP clearing of OTC derivatives. Comparable models of the financial system are built, that reflect the dynamics of the system with central clearing and the bilateral OTC market. This enables us to compare cascading failures and other contagion and default characteristics in both systems. Several network configurations are considered, which function as the basis of our simulation experiments. random networks with and without tiering effects and core-periphery structured networks are recognized and separately considered in the analysis. We examine how concentration, i.e., the number and size of market participants, as well as their capitalization affect system risk in each specific setting. Our analysis takes as a starting point extreme adverse price movements causing a default of at least one financial institution, which potentially can lead to subsequent contagion defaults and possibly a failure of the CCP. From this starting point we investigate how contagion spreads to other parties in a market with central clearing and in a bilateral OTC market.

Our analysis shows that the financial network topology heavily affects the performance of CCPs and their capability of mitigating the system risk. Size differences between clearing members and the existence of "core" (highly connected) players in the financial network determine how contagion spreads. Random networks (which are the least realistic representation of financial networks) show results that are in line with the general hypothesis that CCPs effectively mitigate counterparty credit risk and the limit the spread of contagion losses, compared to bilateral system. Tiered networks, on the other hand, reduce the benefit of CCPs, especially when a large (and highly connected) financial institution defaults. We find that, in these networks, the CCP is unable to reduce contagion risk. This effect is even more pronounced when the so-called core-periphery structure networks are considered (such networks are believed to be 
the most realistic representation of financial systems). In such systems, the contagion risk (and the default probability) increase for smaller financial institutions, when centralized clearing is introduced.

We find that the effects of central clearing for small and large financial institutions are widely different. Large clearing members benefit from it, as risks are shared, while smaller clearing members are disproportionately "sacrificed" in the event of market stress. In all, we find that CCPs can mitigate risks in terms of total capital losses, but not in terms of the number of defaults in the system. Due to contagion, the number of defaulting smaller and less well-connected financial institutions (typically pension and mutual funds) increases with the introduction of CCPs, potentially leading to high social costs. 


\section{Introduction}

System risk and the resulting contagion of losses, especially from OTC derivatives, and its impact on the stability of the financial system became prominent during the 2007-2008 financial crisis. The opaque nature of the OTC derivatives markets combined with mismanagement of risk provided a suitable environment for excessive risk taking by a few institutions leading to major bailouts of these institutions. Various measures have been taken since addressing the issues that are believed to have led to the 2007 financial meltdown. Regulations have been put in place ever since to smooth any disruptive shocks the financial system may face after the default of a Systemically Important Financial Institution (SIFI) and to counter the opaque nature of the OTC derivatives market by central clearing mechanisms capable of monitoring the market. CCPs are burdened with the task of mitigating counterparty credit risk perceived as the principal risk in the financial system and to enforce prudential risk management practices of its GCMs. The financial crisis of 2007-2008 revealed that the bilateral nature of CDS contracts posed a fundamental risk to the system. Enforcement of central clearing will however not apply to all derivatives products as the focus of the recent financial reforms has been on CDSs, as these instruments are held responsible for the initial sub prime crisis and the resulting financial melt down, and interest rate derivatives, being by far the biggest category of OTC derivatives. As far as central clearing of other types of OTC derivatives is concerned, Blundwell-Wignal and Atkinson (2011) note that "this is unlikely to happen for customised structured products, and exemptions will apply for exchange rate derivatives and corporate end-users of derivatives".

As new regulations will mostly apply to already standardized derivatives products in managing systemic risk. This fact may prove to be a challenge for a lot of institutions trading in interest rate derivatives, such as pension funds. Risk sharing mechanisms and margin requirements meant to mitigate counterparty risk is experienced by these parties as an extra source of risk and costs, which might prove difficult to integrate with their business models. The aim of this paper is to contribute in the understanding of contagion risk faced by financial institutions in different network structures. The main objective hereby is to model an OTC derivatives market via application of network theory.

Various approaches in modelling the dynamics of contagion defaults based on topological structures have been proposed in recent literature. Initial research was mostly directed to banking systems from both empirical as theoretical perspectives. Allen and Gale (2000) propose that the completeness of network structures increases system stability. Their claim however, that complete network structures are more robust than incomplete structures, has been criticised and shown to not hold in general. Nier et al. (2008) studies numerically how the structure, interconnectedness and interbank exposures affect the stability of the banking system. Gai and Kapadia (2010) apply statistical techniques to show that financial systems exhibit a robustyet-fragile tendency. A lower probability of contagion is achieved by an increased connectivity. However, the increased connectivity is shown to result in more widely spread contagion in the network, as a result of a higher vulnerability of the system to second-round defaults. Gai et al. (2011) apply network theory to model how systemic liquidity crises similar to the liquidity dry-up in 2007-2008 may be amplified by a greater complexity and concentration. 
Network theory has not been limited to a theoretical framework of analysis. Contagion in financial markets and other cascading failures have been extensively researched by, among others, Furfine (2003), Degryse and Nguyen (2004), Esinger, Lehar and Summer (2006), Lelyveld and Liedorp (2006) and Cont, Moussa and Santos (2012). The general framework of analysis is based on the assumption that contagion defaults are triggered by the default of a single institution within the system. This ensures the tractability of results and differentiates between SIFIs, which affect a considerable number of institutions if not the whole system, and smaller institutions in the sense that they do not lead to widespread contagion. Esinger, Lehar and Summer (2006) criticize this approach in that it only emphasizes the effect of interbank linkage and ignores the correlation between interbank exposures.

A more recent paper by Markose (2012) discusses the spread of contagion within the derivatives market in contrast to most research focused on banking systems. The study shows that a few Systemically Important Financial Intermediaries can lead to considerable losses to the financial system. Markose (2012) notes that most research assumes either networks based on Erdös-Rényi graphs, which are typically random and uncorrelated, and are the main focus of theoretical models, or complete network structures as in Allen and Gale (2000), which are the general setting when empirical data is considered. Markose (2012) argues that a core-periphery network structure captures the true nature of financial markets. Craig and von Peter (2010) and Fricke and Lux (2012) show that this holds for the German bank system and the overnight interbank transactions in the Italian interbank market, respectively. Blundell-Wingel and Atkinson (2011) discuss in detail the challenges faced in derivatives markets due to its sheer size and high concentration.

The ambition to enforce the participation in a system of central clearing for various standardized derivatives products may prove challenging for various market participants. Small asset managers and banks might be more concerned that the risk sharing mechanisms and cost will unevenly punish them. Adverse selection and moral hazard cuts both ways, but its effects on the stability of the system are generally more visible for big firms which makes inferring unbiased conclusions quite hard. It is not our interest however to focus on the such challenges that might be inherent to a system of central clearing. In this paper we are interested in the comparison of stability and the dynamics of a system where OTC derivatives are centrally cleared and one where trades are of bilateral nature. We pose the question how the system risk and its impact on the market participants might manifest in light of a few key parameters. Is the central clearing system more resilient to cascading failures considering the network topology, where distinction is made between a random (tiered) network structure and a tiered core-periphery structure. Secondly, how does the size of the system affect stability. Do more market participant lead to a more stable financial system. And finally, how does a higher buffer affect risk of contagion defaults and losses. Different market participants generally incur losses specific to their business model. In our approach we will be looking at the problem from the perspective of pension funds. This is specifically important when counterparties fail to perform on their obligations. In the rest of the paper we simply refer to these entities as GCMs.

The goal of this paper is to asses how stability and system risk change when moving from OTC derivatives markets to central clearing. 
The remainder of the paper is organized as follows. Section 2 gives a brief description of the OTC derivatives markets and CCPs. We look closely to the some characteristics of the OTC derivatives of our interest. Costs and risks related to central clearing are further discussed in the same section. In section 3 , our model of the derivatives system and the various network topologies is considered. Section 4 contains a brief discussion of the central clearing mechanisms we implement in our model and how they relate to practice. The default dynamics and contagion effects are explained in section 5. Comparative statistics of the simulation experiment and the analyses is given in section 6 . Section 7 concludes. 


\section{OTC derivatives and the realities of central clearing}

The gross notional amount outstanding of OTC derivatives was estimated at $\$ 639$ trillion in the second quarter of 2012 by the Bank for International Settlements (BIS). To put this number into perspective, the world GDP amounted to a total of $\$ 71$ trillion in $2012 .{ }^{1}$ The total notional value of derivatives has witnessed a spectacular growth the past decade attaining an increase of around $600 \%$ in volume size as shown in Figure 2.1. The gross market value and the gross credit exposure are estimated by the BIC at $\$ 25$ trillion (3.9\% of gross notional amount) and $\$ 3.7$ trillion ( $0.6 \%$ of gross notional amount), respectively. These number represent the total OTC derivatives market capitalization. Figure 2.2 shows the split of risk categories in this market with the interest rate derivatives representing the largest category. The size of the interest rate derivatives is estimated around $\$ 494$ trillion at the end of the second quarter of 2012, comprising more than $77 \%$ of the notional amount outstanding. The credit default swap market, criticized for aggravating the global financial crises, only comprised $4.2 \%$ of the notional amount outstanding of OTC derivatives. Figure 2.3 depicts the categorization of interest rate derivatives by currency. The notional amount outstanding of interest rate derivatives at the end of the first quarter of 2012 in Euros and US Dollars equalled $\$ 178,667$ billion and $\$ 164,024$ billion, respectively.

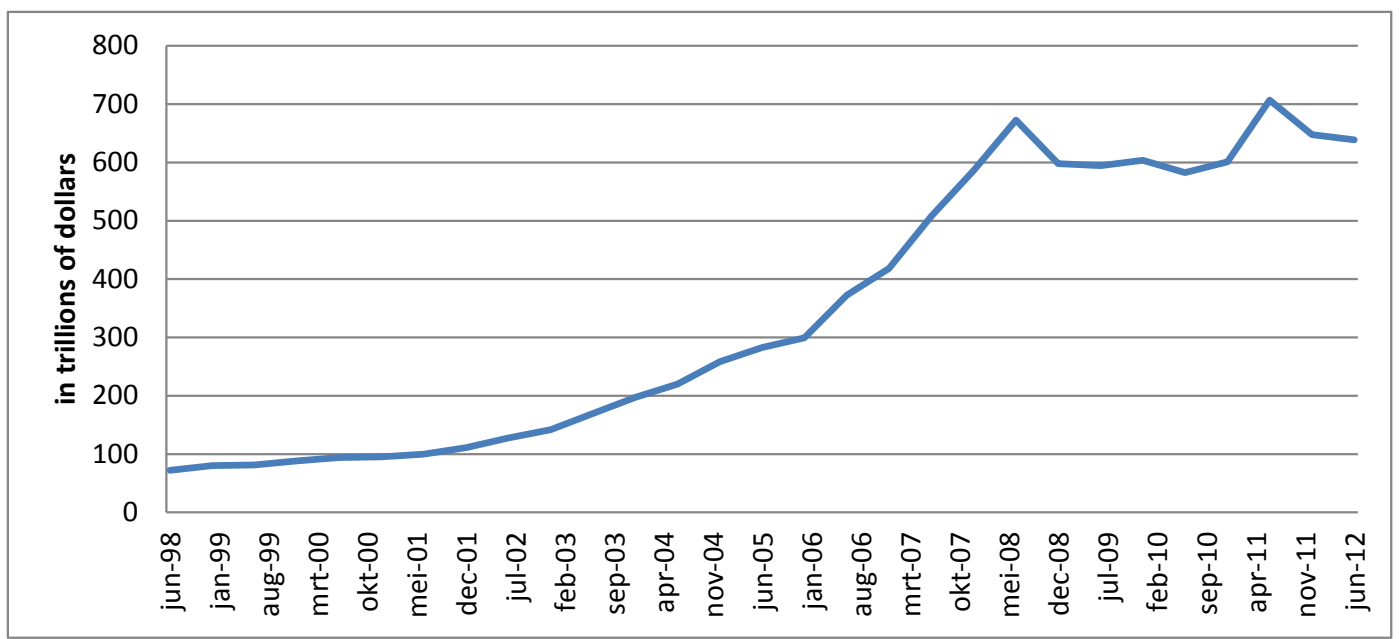

Figure 2.1

Gross notional amount outstanding of OTC derivatives. Source: BIS, November 2012

The sheer size of the OTC derivatives market and the leverage arising from the use of derivatives result in huge revenues and profits, especially for a select few systemically important financial institutions (SIFIs) capable of exploiting the opaque nature of OTC derivatives. However, as the global financial crisis has made clear without a doubt, the other side of the coin is that adverse price movements may easily compromise the financial stability of these institutions as well as the entire financial system. The envisioned centralized clearing counterparties (CCPs) are important components meant to mitigate system risk and to make the opaque OTC market more transparent for market participants and especially regulators. Further, CCPs are

\footnotetext{
${ }^{1}$ IMF World Economic Outlook, Coping with High Debt and Sluggish Growth, October 2012, Table A1
} 


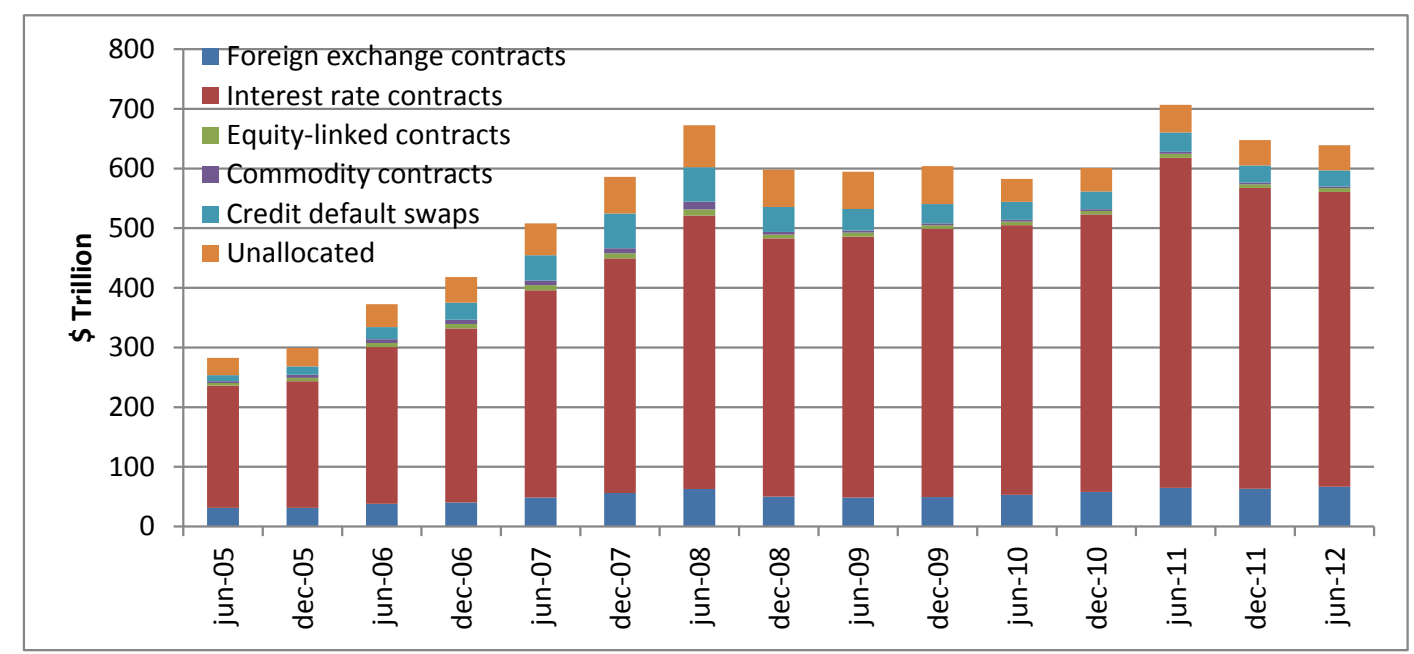

Figure 2.2

OTC derivatives by type. Source: BIS, November 2012

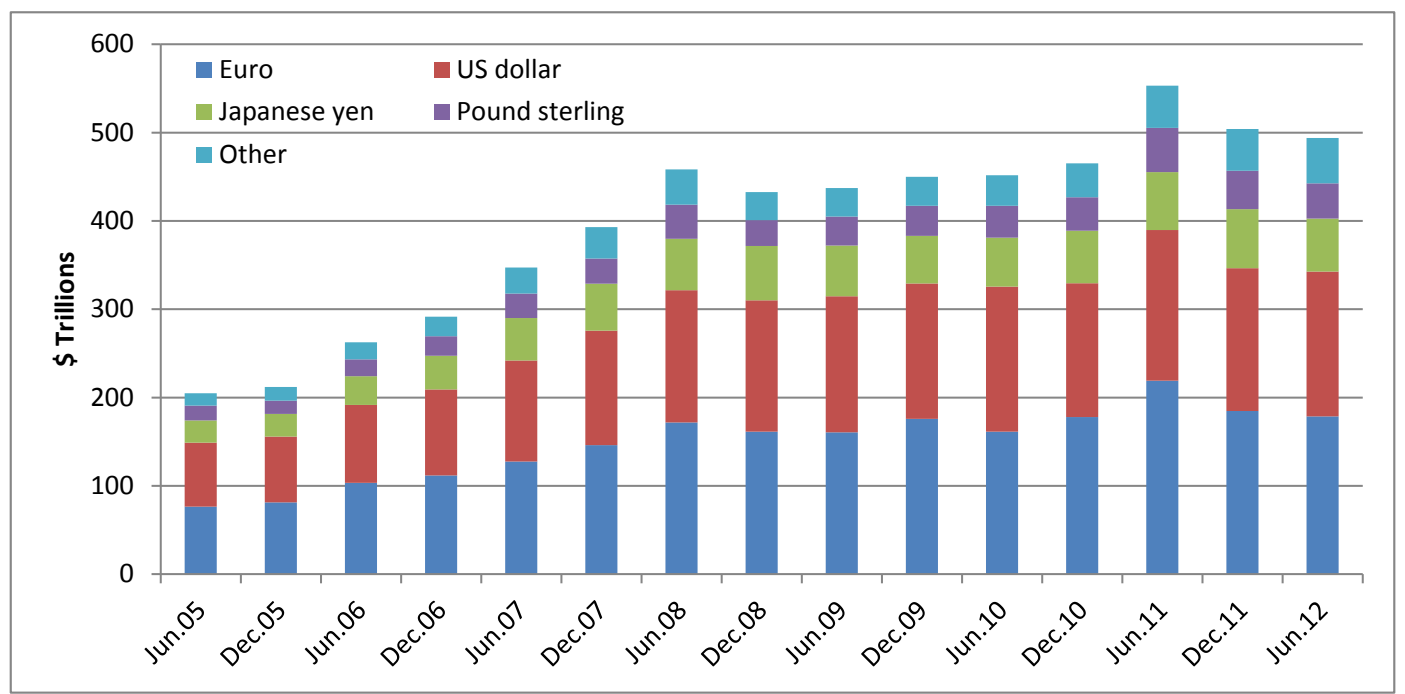

Figure 2.3

Notional amounts of interest rate derivatives by currency. Source: BIS, November 2012

expected to reduce risk exposures among market participants, increase liquidity of the market, reduce transaction fees of each counterparty and improve the efficiency of the capital market. It should be noted however that CCPs are for-profit institutions, thus possibly embodying a inherent weakness in a central clearing system.

CCPs themselves are possible sources of credit risk to their clearing members (GCMs) and hence to the entire financial system. Therefore, it is crucial to improve effectiveness of CCPS' risk control, limit their risk exposures and minimize potential losses in case of defaults of the participants. CCPs manage risks by holding margins and default fund contributions of the clearing members. When a clearing member defaults, these margins and default fund contributions are utilized to cover losses. Whether these losses are incurred by other GCMs depends on the segregation model of the collateral. If the collateral and margins are held in omnibus accounts, then the non-defaulting GCMs are also at risk of having their contributions utilized 
by the CCP. In fact, all GCMs are indirectly exposed to each other through a CCP, so that one GCM's default can lead to the insolvency of other (healthy) members. A CCP maintains zero exposure to GCMs at all times and most, if not all, contracts remain in force after a default. This, combined with one GCM's default, can result in a cascade of defaults of other GCMs. For instance, in case a large GCM defaults, the CCP needs to transfer the defaulter's portfolio, by e.g., auctioning the defaulter's contractual obligations to other GCMs, to keep the CCP hedged. Clearing members are obliged to participate in such an auction or in any other measure the CCP might undertake. This can result in a liquidity pressure and possible market collapse of initially financially healthy clearing members. This is exactly one of the hypothesis that we will test and confirm with our simulations.

Clearing via CCPs comes with additional costs and risks. First of all, these are the additional liquidity costs for the clearing members, arising from the need to post margins and default fund contributions. Some authors argue that, by going from OTC to central clearing of derivatives, the credit risk is essentially replaced by the liquidity risk. Furthermore, central clearing is essentially a protection mechanism where risks are pooled. This kind of risk sharing can create costs in the form of distorted incentives, when information imperfections are present. Moral hazard can arise (or become more prominent) on various levels of the clearing mechanism: on the side of CCPs (e.g., a riskier way of operating if a bailout is assured), and also on the side of GCMs (e.g., "free riding" by GCMs with riskier trade profiles and adverse selection). However, for simplicity, we will not take this moral hazard risk into account in this paper.

Possibly the most pronounced benefit of the central clearing is the increased netting opportunities. This benefit is maximized when a single standardized product is cleared by a single CCP. Moving many different and complex derivatives towards centralized clearing may reduce netting opportunities. Again, for the sake of tractability, we will ignore this effect and assume in our model that a CCP only clears a limited set of highly liquid derivatives.

The pervasive scale and scope effects of CCPs will fundamentally influence the way the clearing sector evolves, having competitive and systemic risk implications. With respect to competitive evolution, scale and scope effects will result in the survival of a small number of large CCPs. CCPs have strong natural monopoly characteristics. It is therefore likely that CCPs will raise anti-trust concerns. This tendency towards the dominance of clearing by a small number of large CCPs will make these entities highly systemically important. The failure of a dominant CCP would have potentially catastrophic effects. Regulatory and legislative interventions that hinder the rise of a few dominant CCPs will, on the other hand, prevent from realizing all risk-reducing benefits of scale. Regulators and legislators will face difficult trade-offs in their oversight of CCPs. Jurisdictional considerations are likely to result in the survival of multiple, under-scaled or under-diversified CCPs. Several major jurisdictions have already made it clear that they will require products traded there (or by firms located in those jurisdictions) to be cleared also there. This jurisdictional fragmentation will prevent market participants from fully taking advantage of the potential scale and scope benefits CCPs can offer. It will also complicate coordination between CCPs, especially in the event of a crisis. Moreover, clearing across jurisdictions poses complicated legal issues, especially insofar as bankruptcy laws are concerned. Again, regulators and legislators (and market participants) will face difficult trade-offs when 
determining the best legal and jurisdictional arrangements for CCPs.

A variety of risk management practices are available to CCPs, such as:

- Initial margin setting. Initial margins are typically determined as a percentage of a derivative's notional. CCPs set and review initial margin levels periodically. Other factors that may affect the necessary amount of collateral requested by a CCP are the size of a clearing member, its risk profile and concentration levels.

- Variation margin setting. CCPs monitor its members' positions continuously, to determine variation margins and risks associated with all the trades. Clearing members are subject to margin calls when the value of their positions deteriorates.

- Default fund contribution. CCPs require their clearing members to contribute to the default fund. The assets in default fund are used in case the initial margin is not enough to bear all the losses due to the default of a clearing member. CCPs will often base default fund contributions on prevailing market conditions and existing margin requirements. A general rule of thumb is that this fund should cover the residual losses of the two largest clearing members.

- Monitoring of clearing members. CCPs also must monitor the financial condition of their members and their operational capability during default. Further, personnel is required to be fully conversant with clearing procedures and capable of evaluating the financial conditions of complex financial entities. CCPs may involve audits in monitoring their clearing members.

- Concentration risk monitoring. Highly concentrated positions pose particularly great risks for CCPs. Clearing members with a highly concentrated position in a product, or a class of related products, may lead to extreme losses in case of adverse price movements. Diversification effects between products become limited and wrong way risk (i.e., the correlation between the exposure and the creditworthiness) becomes more prominent. In such case, defaults are more likely, but also CCPs might face greater difficulty in replacing these concentrated positions or transferring them to the non-defaulting clearing members. Therefore, CCPs will charge higher margins on concentrated positions or restrict certain trades of their clearing members. 


\section{Modelling the financial system}

Our main objective in this paper is to assess whether central clearing mechanisms stabilize the financial system and how they impact the participating parties, i.e., GCMs. To study the contagion of defaults and losses with and without central clearing, our model must capture the characteristic dynamics of the real financial system. When developing our model, we were inspired by several studies that implement a network approach to analyze default cascades in financial systems.

\subsection{Network representations of the financial system}

First, we introduce some notation. The financial system is represented by a numbered set of nodes (financial institutions) $\mathcal{N}=\{1,2, \ldots, n\}$ and the set of possible edges (links between financial institutions) $\mathcal{N} \times \mathcal{N}$. A random graph $\mathcal{E} \subset \mathcal{N} \times \mathcal{N}$ is generated by the function $F(\Theta)$, which we specify later. A realization of $\mathcal{E}$ is represented by the adjacency matrix $A(\mathcal{E})_{(N \times N)}$ :

$$
A=\left[\begin{array}{ccccc}
A_{1,1} & \cdots & A_{1, j} & \cdots & A_{1, n} \\
\vdots & \ddots & \vdots & \ddots & \vdots \\
A_{i, 1} & \cdots & A_{i, j} & \cdots & A_{i, n} \\
\vdots & \ddots & \vdots & \ddots & \vdots \\
A_{n, 1} & \cdots & A_{n, j} & \cdots & A_{n, n}
\end{array}\right]
$$

Loops are not allowed in our system, as these would have zero net effect. So we impose the restriction $A_{i, j}=0$ if $i=j$. Any two nodes $i$ and $j, i \neq j$ linked by an edge in graph $\mathcal{E}$ are represented by $A_{i, j}=1$ and zero otherwise. The system is described by a directed graph, so that each edge has a direction associated with it. If $A_{i, j}=1$ then there is an exposure between parties $i$ and $j$, with $i$ being long and $j$ short in a particular financial position. Note that a cross-directional relation between any two parties is possible by the above definition. Later on, when constructing balance sheets, we will net such positions to retain the net exposure of each party $i$ to counterparty $j$.

The in- and out-degrees of each node represent the number of incoming and outgoing links, respectively. The out-degree $\delta^{+}(i)$ and in-degree $\delta^{-}(i)$ of node $i$ are given by the following expressions:

$$
\begin{aligned}
\delta^{+}(i) & =\sum_{j \in \mathcal{N} \backslash i} A_{i, j}(\mathcal{E}), \\
\delta^{-}(i) & =\sum_{j \in \mathcal{N} \backslash i} A_{j, i}(\mathcal{E}) .
\end{aligned}
$$

The in- and out-degrees enable us to specify the size of each node and determine whether a specific agent is a net creditor $\left(\delta^{-}(i)>\delta^{+}(i)\right)$ or net debtor $\left(\delta^{+}(i)>\delta^{-}(i)\right)$. We characterize the network by weighted links, the weights indicating the total exposures each party has to its 
counterparties:

$$
W=\left[\begin{array}{ccccc}
W_{1,1} & \cdots & W_{1, j} & \cdots & W_{1, n} \\
\vdots & \ddots & \vdots & \ddots & \vdots \\
W_{i, 1} & \cdots & W_{i, j} & \cdots & W_{i, n} \\
\vdots & \ddots & \vdots & \ddots & \vdots \\
W_{n, 1} & \cdots & W_{n, j} & \cdots & W_{n, n}
\end{array}\right]
$$

with

$$
W= \begin{cases}W_{i, j} \geq 1 \quad \text { for } i \neq j \text { and } A_{i, j}=1 \\ W_{i, j}=0 & \text { otherwise }\end{cases}
$$

From (3.5) it follows that $\delta^{+}(i)=\sum_{j \in \mathcal{N} \backslash i} \mathbb{1}_{W_{i, j}>0}$ and $\delta^{-}(i)=\sum_{j \in \mathcal{N} \backslash i} \mathbb{1}_{W_{j, i}>0}$. The way we specify weights is described below.

The paper by Allen and Gale (2001) - one of the earliest works on financial networks - studied financial contagion in complete networks. The study focused on the dynamics of only four fully connected counterparties, whcih represents a so-called complete network. They concluded that a fully connected and homogeneous network results in an increased system stability. Various later works, for instance Markose (2012), have criticized this approach as being unrealistic and thus giving rise to a misleading presumption that a monotonic relationship exist between an increased connectivity and system stability. Many subsequent papers also utilize complete networks and assume (in the absence of information on bilateral positions) that interbank activities are completely dispersed within the system. Upper and Worms (2002) adapt an entropy maximizing algorithm to simulate bilateral exposures in the German interbank market. Degryse and Nguyen (2004) and Lelyveld and Liedorp (2006) similarly conducted their research on the stability of the Belgian and Dutch banking system, respectively.

The next step in complexity from complete networks are random graphs. Erdős and Rényi (1959) was one of the earliest theoretical studies on characteristics of random graphs. In an Erdős-Rényi graph, each edge $l \in \mathcal{N} \times \mathcal{N}$ is present independently of other edges with probability $p$. Hence, the presence of each link in such a graph is governed by the Bernoulli distribution with parameter $p$, resulting in the so-called homogeneous network. The so-called Poisson random graph is a generalization of the standard Erdős-Rényi graph. It is obtained in the following way. Let $n$ be the number of nodes and $p_{n}$ the connection probability, i.e., the probability that $l \in \mathcal{E} \subset \mathcal{N} \times \mathcal{N}$, where graph $\mathcal{E}$ is an arbitrary subset of the set of possible edges $\mathcal{N} \times \mathcal{N}$. Let $p_{n}=\lambda / n$, where $\lambda$ is a constant. Note that $p_{n} \rightarrow 0$ as $n \rightarrow \infty$. Note furthermore that the in-degree $\delta^{-}(i)$ and out-degree $\delta^{+}(i)$ for $0 \leq i \leq n$ are binomial random variables with parameters $n$ and $p_{n}$. It is well-known that under these assumptions the binomial distribution is well approximated by the Poisson distribution. That is, for fixed $x \in \mathbb{N}$,

$$
\left(\begin{array}{l}
n \\
x
\end{array}\right) p_{n}^{x}\left(1-p_{n}\right)^{n-x} \rightarrow e^{-\lambda} \frac{\lambda^{x}}{x !} \text { as } n \rightarrow \infty .
$$

In our study, we will employ Poisson random network, as it is more tractable.

The paper of Nier et al. (2008) performs a numerical study of financial stability and the model employed there is based on random graphs: a homogeneous Erdős-Rényi graph and 
a more realistic model, which introduces a "tiered structure". Banks are divided in 'small' and 'large', where the probabilities of being connected are determined in such a way that large banks have a higher probability of being connected. Gai and Kapadia (2010) use a homogeneous Erdős-Rényi random graph as their model of the financial system and show that such financial systems exhibit robust-yet-fragile tendency. A similar study by Gai et al. (2011) consider two network configurations: a homogeneous random graph and a highly concentrated graph (known as a geometric network). They show that greater complexity and concentration aggravates the spread of a funding contagion.

Some recent studies have found that the financial system has the so-called core-periphery structure (also called a "small world network"), which is not captured by random Erdős-Rényi graphs, even when allowing for tiering or high concentration. Craig and Pieter (2010) find that the so-called block model captures generic relations in a two-tiered network:

$$
\tilde{A}=\left[\begin{array}{ll}
C C & C P \\
P C & P P
\end{array}\right]
$$

The smaller block (subgraph) $C C$ represents highly connected (complete) structure of the subnetwork between the core financial institutions. This subgraph tends to be complete as all institutions in the core are typically linked to one another. Likewise, institutions in the periphery that are unlikely to be linked to other institutions in their class make up the (larger) $P P$ block of the graph. $C P$ and $P C$ blocks represent the relations between financial institutions in the core and the periphery. The adjacency matrix $\tilde{A}$ with $k$ core institutions and $n-k$ institutions in the periphery has the form

$$
\tilde{A}=\left[\begin{array}{ccccccc}
0 & 1 & \cdots & 1 & & \\
1 & 0 & \ddots & \vdots & \tilde{A}_{i, j} \in\{0,1\} \\
\vdots & \ddots & \ddots & 1 & & \\
1 & \cdots & 1 & 0 & & & \\
& & & & 0 & \cdots & 0 \\
\tilde{A}_{i, j} \in\{0,1\} & & \vdots & \ddots & \vdots \\
& & & 0 & \cdots & 0
\end{array}\right]
$$

A typical property of a small world network is a low average path length, meaning that the distance between any two nodes is very small compared to the network size. However, this property alone is not sufficient to characterize small world networks. The short path length property also applies to complete networks by definition (complete networks have the shortest possible average path length possible, i.e., the path length equal to one). This property also holds for random graphs, see Watts and Strogatz (1998). An extra property that sets small world networks apart is related to the so-called clustering coefficient of nodes. Random networks are characterized by a low clustering coefficient, unlike small world networks, where the clustering coefficient remains bounded away from zero. Small world networks are widely used in many applications, for example, they are a perfect model for airline transportation networks, which have a few big and extremely well-connected hubs (such as Chicago O'Hare or Atlanta international airports in the US or Frankfurt airport in Europe). 
Recall that here we are mainly concerned with modelling the financial system from the point of view of the OTC derivatives markets (in particular, interest rate swap markets). From this viewpoint, financial systems, particularly in developed markets, are typical examples of core-periphery, or small-world networks. There are just a few big "core" financial institutions, highly connected to each other (large investment banks such as JPMorgan, Morgan Stanely and GoldmanSachs in the US, Barclays and HSBC in the UK, Deutsche Bank in Germany), which are net sellers of interest rate swaps, while a very large group of (typically smaller) financial institutions, such as pension and mutual funds, insurance companies, are the buyers of interest rate swaps. So our main focus in this paper will be on core-periphery networks, as we believe these represent the typical financial system in the most realistic way. However, there are indications that not all financial systems are small networks. For example, Cont et al. (2010) observe that the Brazilian financial system is not a small world network. So we will also consider in our study standard random graphs (Erdös-Rényi graphs) and their variation with a tiered structure.

\subsection{Constructing random and core-periphery networks}

A random Erdős-Rényi graph is characterized by in-degrees and out-degrees of each node (nodes represent GCMs or counterparties) which are governed by a Poisson distribution in the limit $(n \rightarrow \infty)$ with parameter $\lambda=(n-1) \cdot p, p \in(0,1)$ and network size $n$ of our choosing. A second variation with a tiering structure is considered where nodes are split into two groups, a few nodes of highly connected large GCMs and many nodes of small GCMs with a few connections. Let $\mathcal{L} \subset \mathcal{N}$ be the numbered set of large GCMs and $\mathcal{S} \subset \mathcal{N}$ the numbered set of small GCMs, with $\mathcal{L} \cup \mathcal{S}=\mathcal{N}$ and $\mathcal{L} \cap \mathcal{S}=\oslash$. Further, $|\mathcal{L}|=n_{l}<n_{s}=|\mathcal{S}|$ and $n_{l}+n_{s}=n=|\mathcal{N}|$. To derive a distribution for the simulation of the tiered network structure, we impose the restriction that the total number of links should be equal ex ante. Note that $\delta^{+}(i) \sim \operatorname{Pois}(\lambda)$ for $i \in \mathcal{N}$ holds by definition and thus $\mathbb{E}\left[\delta^{+}(i)\right]=\lambda$. We assumed that the in-degree and out-degree of each node are governed by the same distribution law from which follows that the total number of links in our network equals

$$
\sum_{i \in \mathcal{N}} \mathbb{E}\left[\delta^{+}(i)\right]=n \cdot \lambda
$$

as $\delta^{+}(i)$ are identically independent distributed variables $(\mathcal{I} \mathcal{I D})$. Looking back at the tiered network structure where the $\delta^{+}(i)$ need to be specified separately for each node class. We want to connect our network in such a way that the total number of links is equal to 3.8. This ensures that the simulation result are comparable in both settings. We keep the assumption in the tiered network structure that $\delta_{l}^{+}(i) \sim \operatorname{Pois}\left(\lambda_{l}^{+}\right)$and $\delta_{l}^{-}(i) \sim \operatorname{Pois}\left(\lambda_{l}^{-}\right)$for $i \in \mathcal{L}$ are similarly distributed. Likewise, $\delta_{s}^{+}(i) \sim \operatorname{Pois}\left(\lambda_{s}^{+}\right)$and $\delta_{s}^{-}(i) \sim \operatorname{Pois}\left(\lambda_{s}^{-}\right)$for $i \in \mathcal{S}$ so that $\lambda_{l}^{+}=\lambda_{l}^{-}=\lambda_{l}$ and $\lambda_{s}^{+}=\lambda_{s}^{-}=\lambda_{s}$. Let $p_{l}>p$ be the probability that a link is extant from a large GCM to an arbitrary GCM and $p_{s}<p$ the probability of a link from a small GCM to an arbitrary GCM. Thus, $\lambda_{l}=(n-1) \cdot p_{l}$ and $\lambda_{s}=(n-1) \cdot p_{s}$ by definition. Finally, using the fact that $\delta^{+}(i) \sim \mathcal{I} \mathcal{I D}$ 
and that 3.8 must hold, we must solve the following equation for $p_{l}$ and $p_{s}$ :

$$
\begin{aligned}
\sum_{i \in \mathcal{L}} \mathbb{E}\left[\delta_{l}^{+}(i)\right]+\sum_{i \in \mathcal{S}} \mathbb{E}\left[\delta_{s}^{+}(i)\right] & =\sum_{i \in \mathcal{N}} \mathbb{E}\left[\delta^{+}(i)\right] \\
\Rightarrow l \cdot \lambda_{l}+s \cdot \lambda_{s} & =n \cdot \lambda \\
\Rightarrow l \cdot(n-1) \cdot p_{l}+s \cdot(n-1) \cdot p_{s} & =n \cdot(n-1) \cdot p
\end{aligned}
$$

This equation of two unknowns is solved for a fixed $\tilde{p}_{l} \in(0,1)$ :

$$
\tilde{p}_{s}=\frac{n \cdot p-l \cdot \tilde{p}_{l}}{s}, \tilde{p}_{l}>p .
$$

To wire our graph we perform the following steps ensuring the right distribution of the inand out-edges.

\section{Algorithm 1: For a fixed set of parameters $\Theta=\left\{n, l, p, \tilde{p}_{l}, \tilde{p}_{s}\right\}$}

(i) Draw n independent samples $\left(\bar{\delta}^{+}(i), \bar{\delta}^{-}(i)\right), \forall i \in \mathcal{L} \cup \mathcal{S}$.

(ii) The network is wired by taking out for each node $i, \bar{\delta}^{+}(i)$ and randomly wiring it with the remaining unpaired nodes $j \neq i$.

(iii) A loop for $i=1, \ldots, n$ results in that all nodes are connected.

Figure 3.1(a) and 3.1(b) show an example of a random homogeneous network and a network with a tiering structure, respectively. Both networks consist of $n=25$ nodes and $p$ is kept fixed at 0.2. Equation 3.9 is solved for $\tilde{p}_{l}=0.5$ and $l=\lceil 0.1 \cdot n\rceil$ to obtain the network given in 3.1(b). The size of each node is determined based on the average number of edges. This average is obtained from the in- and out-degree of each node: $\frac{\delta_{s}^{+}(i)+\delta_{s}^{-}(i)}{2}$. A completely random network has homogeneous nodes (ex ante) as the distribution of $\delta_{s}^{+}(i)$ and $\delta_{s}^{-}(i)$ is the same. In contrast, a tiered network structure has $l$ large nodes and $s=n-l$ smaller ones (ex ante). This effect is not clearly visible for the simulated networks in figure 3.1 as $n$ is quite small. We can remark that more nodes appear to be of similar size in figure 3.1(a), where the number of 'large' nodes (the larger the bluer a node) and small nodes (the smaller the redder a node) appears to be divided evenly. Figure 3.1(b) on the other hand shows a network were a fewer large nodes and many smaller nodes are present. We would expect for $n=25$ to obtain 3 clearly large nodes and 22 smaller ones, which appears to be true more or less. To get a clearer picture of this fact, we increase the size of the network by taking $n=100$. A simulated random network and a network with tiering is shown in figure 3.2. 3.2(a) shows that the node sizes are more or less of comparable size with a few exceptions since the in- and out-degrees are stochastic. Figure 3.2 clearly shows to types of nodes, the $10(0.1 \cdot n)$ larger and the remaining 90 smaller ones, unlike the homogeneous network.

\subsubsection{Core-Periphery Structure}

The second type of network we consider in our simulations is a core-periphery structured network. The blockmodel given in 3.6 capture the generic relations in a two tiered network. Our aim is to simulate a network taking into consideration some restriction we impose particularly on the size of the financial system. We want this to be kept fixed in all cases, keeping in mind 


\section{Figure 3.1}

Erdős-Rényi Graphs: The simulated networks consist of $n=25$ nodes and the wiring probability $p$ is kept fixed for both the random network (left) and the random tiered network (right).

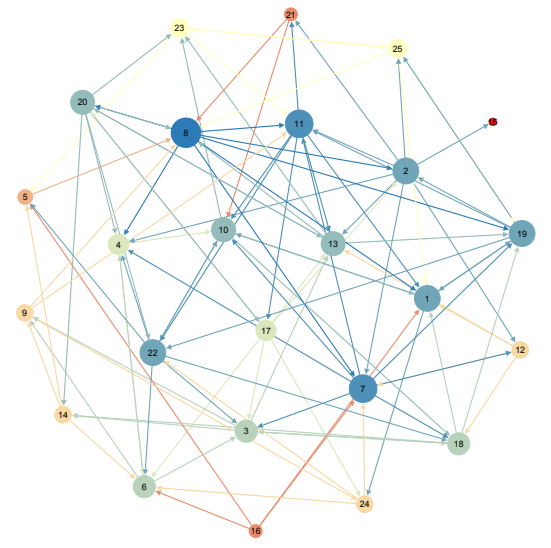

(a) Random: $p=0.2$

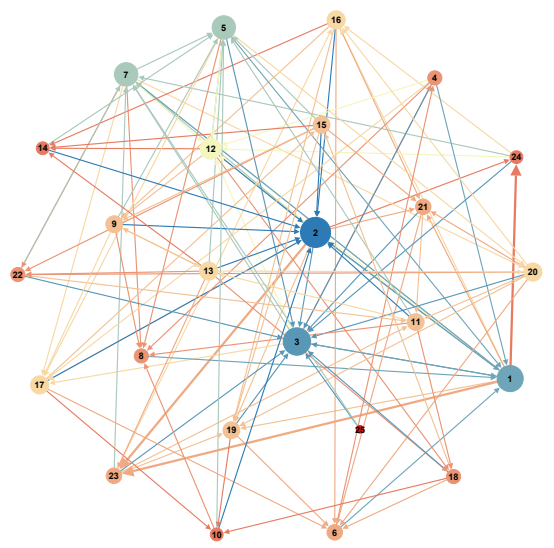

(b) Tiered Structure: $p_{l}=0.5, p_{s}=0.16$

\section{Figure 3.2}

Erdös-Rényi Graphs: The simulated networks consist of $n=100$ nodes. The wiring probability is kept at $p=0.2$ to make comparison with the smaller networks $(n=25)$ possible.

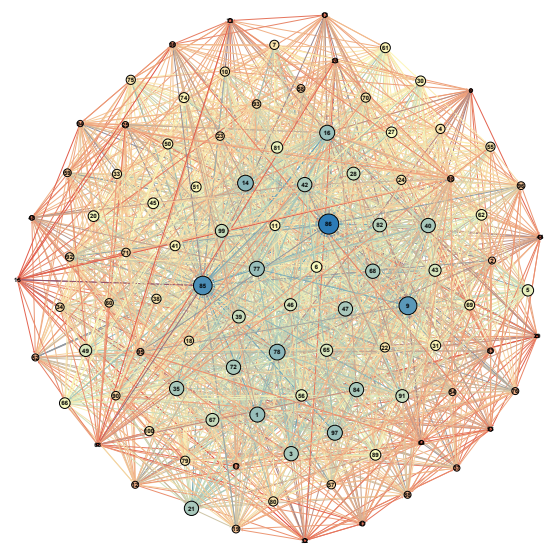

(a) Random $p=0.2$

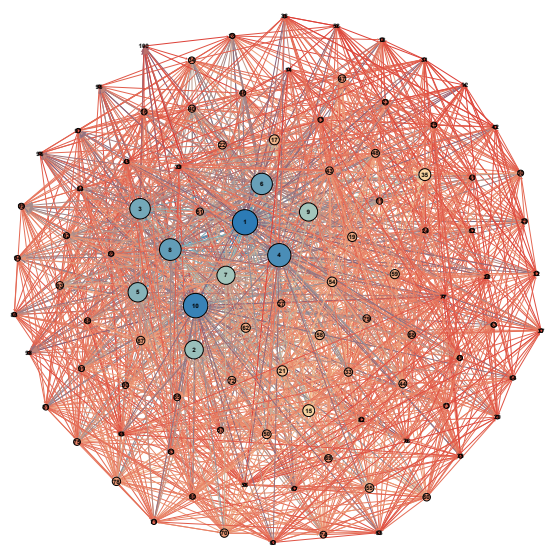

(b) Tiered Structure $p_{l}=0.5, p_{s}=0.17$

that each link represents a cash flow or mutual exposures the GCMs face. We achieved this for the random network by equation 3.8. Opposite to this approach, we now impose the restriction that the total weight of all links, as given in equation 3.4, should ex-ante be the same for all networks. Further, GCMs in the periphery are not allowed to be connected to each other as mentioned earlier. Finally, an extra restriction is needed to ensure that large GCMs control a certain fraction $x$ of the total market. This is achieved by setting up equation 3.10 and 3.11 and solving for $p_{l}$ and $p_{s}$ which can be uniquely determined given the fact that we have two 
equations and two unknowns.

$$
\begin{aligned}
p \cdot n \cdot(n-1) & =p_{l} \cdot l \cdot(n-1)+p_{s} \cdot s \cdot(n-1) \\
p_{l} \cdot l \cdot(n-1) & =\frac{x}{1-x} \cdot p_{s} \cdot s \cdot(n-1) \\
\Rightarrow p_{l} & =\frac{p \cdot n \cdot x}{l} \\
\Rightarrow p_{s} & =\frac{p \cdot n \cdot(1-x)}{s}
\end{aligned}
$$

The parameters $n, l$ and $p$ for number of GCMs, number of large GCMs and the probability of occurrence of every possible edge as given for the Erdös-Rényi graph, respectively, are kept fixed to make all networks comparable. Equation 3.10, which was derived in the previous section, ensures that the expected number of links in the random Erdös-Rényi network equals the total weight in the core-periphery tiered network. We ascertain that the large and small GCMs make up the right fraction of the total market size by equation 3.11 .

We adjust the wiring algorithm in such a way that all these restrictions are satisfied in the following way:

\section{Algorithm 2: For a fixed set of parameters $\Theta=\left\{n, l, p, \tilde{p}_{l}, \tilde{p}_{s}\right\}$}

(i) Draw n independent samples $\left(\bar{\delta}^{+}(i), \bar{\delta}^{-}(i)\right), \forall i \in \mathcal{L} \cup \mathcal{S}$.

(ii) Nodes are numbered and ranked so that

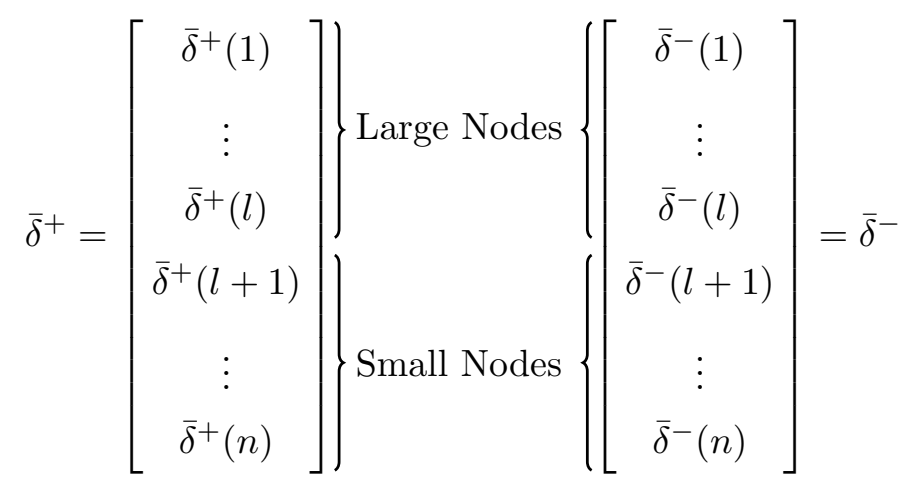

(iii) Take a free out-stub $\bar{\delta}^{+}(i)$ for $i>l$ and randomly wire it to a node with a free in-stub $\bar{\delta}^{-}(j)$ for $j \leq l$ until $\bar{\delta}^{+}(i)=0$ for $i>l$.

(iv) Repeat the previous step but now by coupling the free in-stubs $\bar{\delta}^{-}(i)$ for $i>l$ with a random out-stub $\bar{\delta}^{+}(i)$ for $i \leq l$ until $\bar{\delta}^{-}(i)=0$ for $i>l$.

(v) Finally, all remaining nodes are randomly linked to each other until either $\bar{\delta}^{+}=\mathbf{0}$ or $\bar{\delta}^{-}=\mathbf{0}$. 


$$
\left.\left[\begin{array}{c}
\bar{\delta}^{+}(1) \\
\vdots \\
\bar{\delta}^{+}(l) \\
\bar{\delta}^{+}(l+1) \\
\vdots \\
\bar{\delta}^{+}(n)
\end{array}\right]\right\}\left\{\begin{array}{c}
\bar{\delta}^{-}(1) \\
\vdots \\
\bar{\delta}^{-}(l) \\
\bar{\delta}^{-}(l+1) \\
\vdots \\
\bar{\delta}^{-}(n)
\end{array}\right]
$$

Figure 3.3(a) and 3.3(b) show an instance of a tiered random network and a core-periphery tiered network, respectively. Figure 3.1(b) is duplicated in figure 3.3(a) to make the difference between random networks and core-periphery structured networks distinct. Once more, the network consists of $n=25$ nodes, representing 25 GCMs. As mentioned before, the random tiered network is constructed by keeping $p=0.2$ fixed and solving equation 3.9 for $\tilde{p}_{l}=0.5$ and $l=\lceil 0.1 \cdot n\rceil$. The core-periphery tiered network is constructed by solving equation 3.10 and equation 3.11 for $p=0.2, x=0.8, l=\lceil 0.1 \cdot 25\rceil=3$, from which we obtain $\tilde{p}_{l}$ and $\tilde{p}_{s}$. Large GCMs in the core are much clearly distinguishable from the small GCMs in the periphery as can be noticed from figure 3.3(b) compared to 3.3(a). We notice that the different node sizes representing the large GCMs and the small GCMs are much more distinct even for small networks. Likewise, the linkage between the different nodes is much orderly. Small GCMs are mostly connected to only one core GCM and do not have any connections mutually unlike large GCMs satisfying the blockmodel given by equation 3.6. This effect is even more clearly

\section{Figure 3.3}

Core-Periphery Structure: The simulated networks consist of $n=25$ nodes. Equation 3.9, 3.12 and 3.13 are solved for the same wiring probability $p$ to make both graphs comparable.

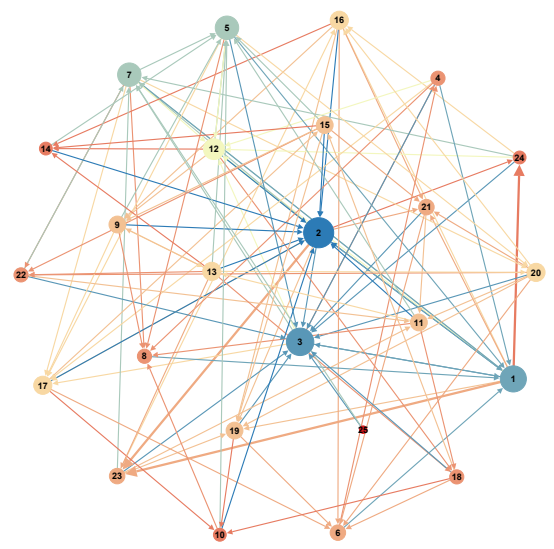

(a) Tiered Structure

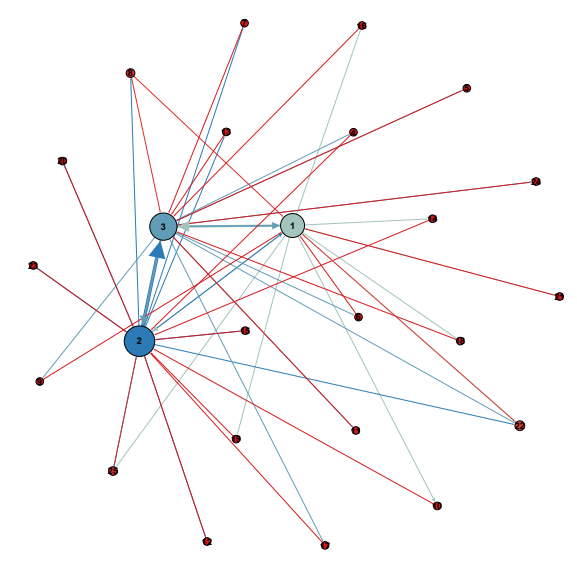

(b) Core-Periphery Structure

visible when considering a larger network with more nodes as shown in figure 3.4. Once again, figure 3.2(b) is duplicated in 3.4(a) to make the difference between random networks and coreperiphery structured networks distinct. Large GCMs have a higher connectivity to each other in figure 3.4(b) compared to the network in 3.4(a). In contrast, small GCMs in the periphery are only connected to GCMs in the core in 3.4(b) unlike the ones in 3.4(a). Finally, the size 
difference is more persistent in the core-periphery tiered network in figure 3.4(b) compared to the tiered random network in 3.4(a).

\section{Figure 3.4}

Core-Periphery Structure: The simulated networks consist of $n=100$ nodes. Equation 3.9, 3.12 and 3.13 are solved for the same wiring probability $p$ to make both graphs comparable.

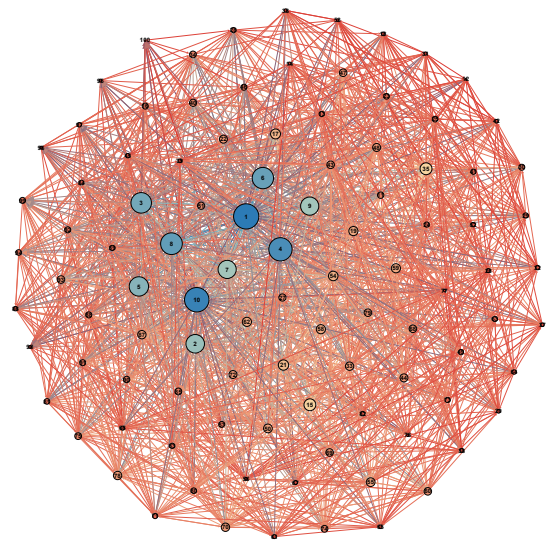

(a) Tiered Structure

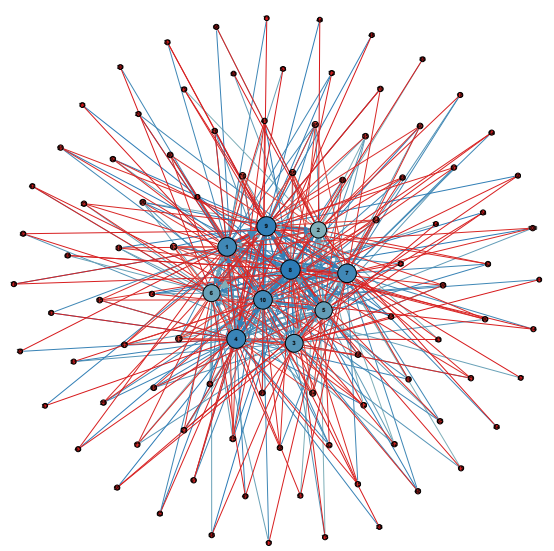

(b) Core-Periphery Structure

\subsection{Balance sheets}

In order to study the impact of central clearing of OTC derivatives we need a simple representation of the GCMs and consequently the derivatives market as a whole. This is achieved by modifying the proposed model of a banking system by Nier et al. (2008) to satisfy the characteristics of our system. The balance sheets are then simulated in a consistent manner, for each realisation of our network, as to ensure that the balance sheet identities and aggregate balance sheet identities are satisfied. Our main objective herein is to determine the assets and capital each GCM has available. Assets can be posted as collateral and capital functions as buffer for GCMs if any losses arise. Likewise, the portfolio mix of each GCM that will be the basis of our simulation experiment needs to be defined. Any losses arising in our system are caused by adverse 'price' movements leading to losses for these portfolios.

As mentioned earlier, the notional amount outstanding of interest rate derivatives was estimated to be around 7 times the size of the world GDP by the BIS. This size difference is taken into account by first deciding on the size of the interbank system. By the interbank system we refer to the cash flows between the GCMs which directly follow from the simulated links in our networks.

An individual GCM's assets, denoted by $A$, include the fixed assets $A^{F}$, liquid assets $A^{L}$, and the interbank assets $A^{I A}$. We do not make any distinction between fixed and liquid assets, to which we will simple refer to as foreign assets in short, and simply assume that GCMs have recourse to these assets for margin calls when the need arises. The interbank assets are assumed to be secured and hence do not lead to any aggravation of losses if a GCM defaults, i.e. fails to perform on its contractual obligations towards the $\mathrm{CCP}$ or its counterparties in a bilateral OTC 
market, within the interbank system as we are only interested in the default dynamics within the OTC derivatives market. Thus, for GCM $i$, we have $A_{i}=A_{i}^{F}+A_{i}^{L}+A_{i}^{I A}$, for $1 \leq i \leq n$.

An individual GCM's liabilities, denoted by $L$, include the GCM's capital $L^{C}$ (which we will refer to simply as $c$ in later sections), deposits $L^{D}$ and interbank liabilities $L^{I L}$. Thus, for GCM $i$, we have $L_{i}=L_{i}^{C}+L_{i}^{D}+L_{i}^{I L}$, for $1 \leq i \leq n$. Obviously, for all GCMs it holds that $A_{i}=L_{i}$ as the two sides need to balance out. A stylized balance sheet is given in table 3.1.

\begin{tabular}{l|l} 
Assets & Liabilities \\
\hline Fixed Assets $A_{i}^{F}$ & Capital $L_{i}^{C}$ \\
\hline Liquid Assets $A_{i}^{L}$ & Deposits $L_{i}^{D}$ \\
\hline Interbank Assets $A_{i}^{I A}$ & Interbank Liabilities $A_{i}^{I L}$ \\
\hline
\end{tabular}

Table 3.1

Stylized balance sheet of an individual GCM

All balance sheet components are determined endogenously depending on the simulated network except for the total size of foreign assets $A^{F L}=A^{F}+A^{L}=\sum_{i=1}^{n}\left(A_{i}^{F}+A_{i}^{L}\right)$ which we use to initialize the construction of the balance sheets. Our first step is thus to decide on the total size of the aggregated foreign assets in the system which we arbitrarily fix at an amount $\Xi \in \mathbb{N}$. All our results are reported in relative terms so that any specific choice of $\Xi$ has no significance.

Next, the size of the interbank system $A^{I A}$ is determined as a percentage of the total aggregated foreign assets. By definition we have

$$
A^{I A}=\sum_{i=1}^{n} A_{i}^{I A}=\sum_{i=1}^{n} L_{i}^{I L}=L^{I L} .
$$

Note that $A_{i}^{I A}=L_{i}^{I L}$ does not have to hold. We determine $A^{I A}$ as a percentage $\Theta_{I}$ of $\Xi$, the aggregated amount of foreign assets, with $\Theta_{I} \leq 0.5$. The interbank assets and liabilities of individual GCMs are then determined in a straightforward manner by considering any arbitrary network. As mentioned earlier, each network is described by its adjacency matrix as given in equation 3.1 and equation 3.7 for a random and a core-periphery structured network, respectively. The weights of each link are consequently captured by the weight matrix $W$ in equation 3.4. Dividing $A^{I A}$ by the sum of all the entries of the weight matrix $W$ gives us the size $w$ of the cash flow of any link with a weight equal to 1 as given in equation 3.14 .

$$
w=\frac{A^{I A}}{\sum_{i=1}^{n} \sum_{j=1}^{n} W_{i, j}} .
$$

The interbank assets and liabilities of each individual GCM are then determined as follows:

$$
\begin{aligned}
A_{i}^{I A} & =w \cdot \sum_{j=1}^{n} W_{i, j} \\
L_{i}^{I L} & =w \cdot \sum_{j=1}^{n} W_{j, i}
\end{aligned}
$$


The foreign assets of each GCM is the next balance sheet component we determine from the interbank assets, liabilities and $\Xi$. We require the foreign assets to be no less than the net interbank assets, meaning $A_{i}^{F L} \geq \max \left\{0, L_{i}^{I L}-A_{i}^{I A}\right\}$. This is achieved in two steps. Our first step is to increase $A_{i}^{F L}$ for all GCMs who's lending exceeds its borrowing so that the balance sheets balances out:

$$
\tilde{A}_{i}^{F L}=\left\{\begin{aligned}
L_{i}^{I L}-A_{i}^{I A} & \text { if } A_{i}^{I A} \leq L_{i}^{I L}, \\
0 & \text { otherwise }
\end{aligned}\right.
$$

The second step is to distribute the remaining $\tilde{\Xi}=\Xi-\sum_{i=0}^{n} \tilde{A}_{i}^{F L}$ among all GCMs. In other words,

$$
\tilde{\tilde{A}}_{i}^{F L}=\frac{\tilde{\Xi}}{n} .
$$

We adjust the way we allocate $\tilde{\Xi}$ for the core-periphery tiered network as to not diminish the difference in size between the large GCMs and small ones in the following way:

$$
\tilde{\tilde{A}}_{i}^{F L}=\left\{\begin{aligned}
x \frac{\tilde{\Xi}}{l} & \text { for large GCMs } \\
(1-x) \frac{\frac{\Xi}{s}}{} & \text { for small GCMs }
\end{aligned}\right.
$$

This adjustment better reflects the size difference between GCMs, which further affects the remaining components of the balance sheet we still need to determine. The foreign assets of each GCM are thus equal to

$$
A_{i}^{F L}=\tilde{A}_{i}^{F L}+\tilde{\tilde{A}}_{i}^{F L} .
$$

A solution for the first step is always guaranteed to exist because of the restriction $\Theta_{I} \leq 0.5$ introduced earlier.

The steps above completely determine the assets side of the balance sheet. The last two components of the balance sheets on the liability side are obtained from the asset side in a straightforward manner. The capital of each GCM is assumed to be a fixed proportion of the asset side. Let $\Theta_{\gamma} \in(0,1)$ be the net worth of any GCM (We keep $\Theta_{\gamma}$ fixed for all GCMs) so that

$$
L_{i}^{C}=\Theta_{\gamma} \cdot\left(A_{i}^{F L}+A_{i}^{I A}\right),
$$

for $1 \leq i \leq n$. The deposits $L_{i}^{D}$ are finally determined as a balancing component so that

$$
\begin{aligned}
& A_{i}^{F L}+A_{i}^{I A}=L_{i}^{C}+L_{i}^{D}+L_{i}^{I L} \\
\Rightarrow & L_{i}^{D}=A_{i}^{F L}+A_{i}^{I A}-L_{i}^{C}-L_{i}^{I L},
\end{aligned}
$$

for $1 \leq i \leq n$. This completes the construction of all components of the balance sheet.

\subsubsection{Portfolio Mix}

The next step is to determine the portfolio mix of each individual GCM. The balance sheets were simulated in such a way that they reflect the relative size of the GCMs by determining the 
interbank liabilities and assets based on the number of connections, their weight and their size class as given in equation 3.15, 3.16 and 3.17, respectively. Hence we can infer the relative size of the portfolio of any given GCM by taking into account the relative size of its assets.

We assume that $M$ types of contracts are traded in our system. Each GCM hold a certain number of contracts of each type relative to its own relative size within the system, say $\Delta_{i, m}$ for $i=1, \ldots, n$ and $m=1, \ldots, M$. Hence, each GCM holds $\Delta_{i}=\sum_{i=1}^{M} \Delta_{i, m}$ contracts in total. Further, each contract consists of two legs, a floating leg and a fixed leg, since we will focus specifically on interest rate swaps (IRSs). Furthermore, we assume that a GCM does not hold both a floating leg and a fixed leg at the same time of a particular contract. The changes in prices would otherwise cancel each other out. Hence, the portfolio of each GCM $i$ is described by $\Delta_{i, m, k}$ for $i=1, \ldots, n, m=1, \ldots, M$ and $k \in \mathcal{F}=\{$ Floating, Fixed $\}$.

Our aim is to determine $\Delta_{i, m, k}$ in such a way that all the characteristics above are satisfied. We first discuss the case where our network is completely random as is the case for Erdős-Rényi graphs. All GCMs are in this case ex-ante of equal size. Each GCM holds a specific number of contracts $\Delta_{i, m, k}$, which we decide on arbitrarily. Next we assume that each GCM has a $50 \%$ chance of holding a fixed leg or floating leg of each specific contract, i.e. we select a set of GCMs holding the fixed leg and the rest holds the floating leg. The last step is then to decide on the counterparties. This is done by randomly selecting for each GCM another GCM that functions as counterparty. The last selection step is of more importance when a bilateral OTC market is simulated, which is used to compare the result when central clearing is introduced. Later on we will see that keeping track of the counterparties can be useful when a CCP fails and the need arises to go back to a bilateral OTC market.

The procedure above is slightly adjusted to take size differences of the GCMs in tiered networks into account. The total number of contracts in the whole system equals

$$
\Delta=\sum_{i=1}^{n} \sum_{m=1}^{M} \sum_{k \in \mathcal{F}} \Delta_{i, m, k} .
$$

We allocate $\Delta_{L}=x \Delta$ to the large GCMs and $\Delta_{S}=(1-x) \Delta$ to the rest. $\Delta_{L}$ and $\Delta_{S}$ are then equally divided over the $M$ contract types. This gives us the right $\Delta_{L, i, m, k}$ for the large GCMs and $\Delta_{S, i, m, k}$ for the rest.

Each portfolio has a value that needs to be modelled. This is achieved by summing up the values of all contract held by a GCM. As it is not our aim to dwell to much on this subject, we opt for a simple model to obtain a starting point for our simulation experiment. The value of each IRS contract for a counterpartie with a long position, paying floating and receiving fixed, is the difference between the value of both legs represented by bonds.

$$
V_{t, f i x, m}=B_{t, f i x, m}-B_{t, f l, m}
$$

where $B_{t, f i x, m}$ and $B_{t, f l, m}$ stand for the value of the fixed leg and floating leg at time $t \geq 0$, respectively. With a slight abuse of notation, $V_{0}^{G}$ stands for the gross value of an IRS and $V_{i, t}$ the value of the portfolio of GCM $i$ at time step $t$. Likewise, the value of the IRS contract for 
a party with a short position, paying fixed and receiving floating, is the difference between the value of the floating leg and fixed leg, thus $V_{t, f l, m}=-V_{t, f i x, m}$. The value of swap $m$ is then given as a function of $y_{t, m}$, the yield at time $t$ :

$$
V_{t, f i x, m}=f\left(y_{t, m}\right)
$$

Lastly we compute the price changes by considering the (modified) duration $D^{*}$ as follows:

$$
\Delta B=-B D^{*} \Delta y
$$

The last step in our modelling is to model the trading exposure between the $n$ counterparties and the cleared exposures the CCP faces. For the sake of simplicity, we assume that each of the $M$ IRSs can be modelled by a their own Wiener process $W_{t, m}$,

$$
\mathrm{d} r_{t}=\hat{\mu}_{m} \mathrm{dt}+\hat{\sigma}_{m} \mathrm{dW}
$$

for $i=1, \ldots, M$. Our approach is to model the price jump of each contract at time $t$ given the duration of each contract, the tenor $T_{m}$ and yield $y_{0, m}$ for $m=1, \ldots, M$. Given the returns $r_{t}$, for $t \geq 0$, we calculate the yields $y_{t}$ as

$$
y_{t}=y_{t-1} e^{r_{t}}
$$

for $t \geq 1$, from which we obtain

$$
\Delta y_{t}=y_{t}-y_{t-1}
$$

The initial margins, variation margins and default fund contribution can be derived from the swap contracts value at any time $t>0$. The initial margins are determined as fixed contribution $\Theta_{\alpha}$ a GCM has to make for each contract it hold. Thus, the total funds GCM $i$ has to deposit in the margin account is given by

$$
\Theta_{\alpha} V_{0}^{G}\left(\sum_{m=1}^{M} \sum_{k \in \mathcal{F}} \Delta_{i, m, k}\right)=\Theta_{\alpha} V_{t}^{G}\left(\Delta_{i}\right),
$$

Where $V_{t}^{G}$, at $t=0$, is the gross notional value of the contracts at initiation. Since all contracts have the same face value, we do not need to differentiate between specific contracts. We only need to know how many contracts each party holds to determine the initial margins.

The default fund contribution is trickier to determine. Since we want to keep our model as simple as possible, we opt for a straightforward approach. The default fund contribution

$$
f\left(\Theta_{\beta}, \Theta_{\alpha}, V_{t}^{G}(\cdot)\right)
$$

is a function of both the initial margin contribution $\Theta_{\alpha}$ and the gross notional value of the portfolio. The specific form is given later on.

Finally, the variation margins are the extra funds a GCM has to deposits when it faces negative price movements. These are determined from the swap value given in equation 3.21 at each time step $t>0$ and can either be positive or negative depending on the portfolio value. 
Lastly, the complete balance sheet is given in table 3.2, where the margins are taken into account by a separate account. That is, to take into account the fact that these funds are actually not available to the GCM in case of default or when a CCP utilizes these assets to cover for residual losses. Counterparties in a bilateral OTC market on the other hand do have access to these assets. Furthermore, these parties do not need to contribute to a default fund and do not face the risk of having there assets utilized to cover for residual losses of contagion defaults for instance.

\begin{tabular}{l|l} 
Assets & Liabilities \\
\hline Fixed Assets $A_{i}^{F}$ & Capital $L_{i}^{C}$ \\
\hline Interbank Assets $A_{i}^{I A}$ & Interbank Liabilities $L_{i}^{I L}$ \\
\hline Liquid Assets $A_{i}^{L}$ & Deposits $L_{i}^{D}$ \\
\hline Margins $M_{i}$ & \\
\hline
\end{tabular}

Table 3.2

Stylized balance sheet of an individual GCM 


\section{Central Clearing Mechanisms}

Our aim in this paper is to assess the impact on the financial system of clearing of OTC derivatives in extreme scenarios. CCPs aim to not only reduce the likelihood of financial crises but also reduce contagion by employing several requirements for GCMs and risk management methods. These safeguards are meant to not only protect GCMs but also to avoid any spillover of losses to the system. We stress that it is not our aim to address the issue in how far CCPs are able to mitigate risks and avoid defaults by means of their risk management methods. The main focus of this paper is to compare how losses will affect the system in extreme scenarios and how far the risk sharing mechanisms are capable of softening the losses to the financial system or if they might end up aggravating these losses in these extreme scenarios. For instance, SwapClear, an LHC.Clearnet global clearing service for IRSs and currently clearing more than $50 \%$ of the global notional market, applies a number of layers of protection in a default scenario to meet the obligations of the defaulter. A so called Default Waterfall, as shown in figure 4.1, is set up to cover for any losses and to ensure the performance of cleared IRSs. CCPs will request margin (collateral) to cover potential losses if a member would default. Variation margin are requested when the net present value of the outstanding trades moves in such a way that the initial margins are not sufficient to cover the exposure. The default fund contribution is the next protection layer meant to cover any losses not covered by the margin calls. Finally, the CCPs capital is utilized if needed as a final measure to cover any residual losses. In the remainder of this chapter we treat some of these risk sharing mechanisms and risk management methods and their application in our model. We discuss in short how we apply the default fund contribution in our model. Calculations for the variation margins were given in the previous chapter. We expand on how we decide on the parameters of equation 3.23 and equation 3.24. Further, we

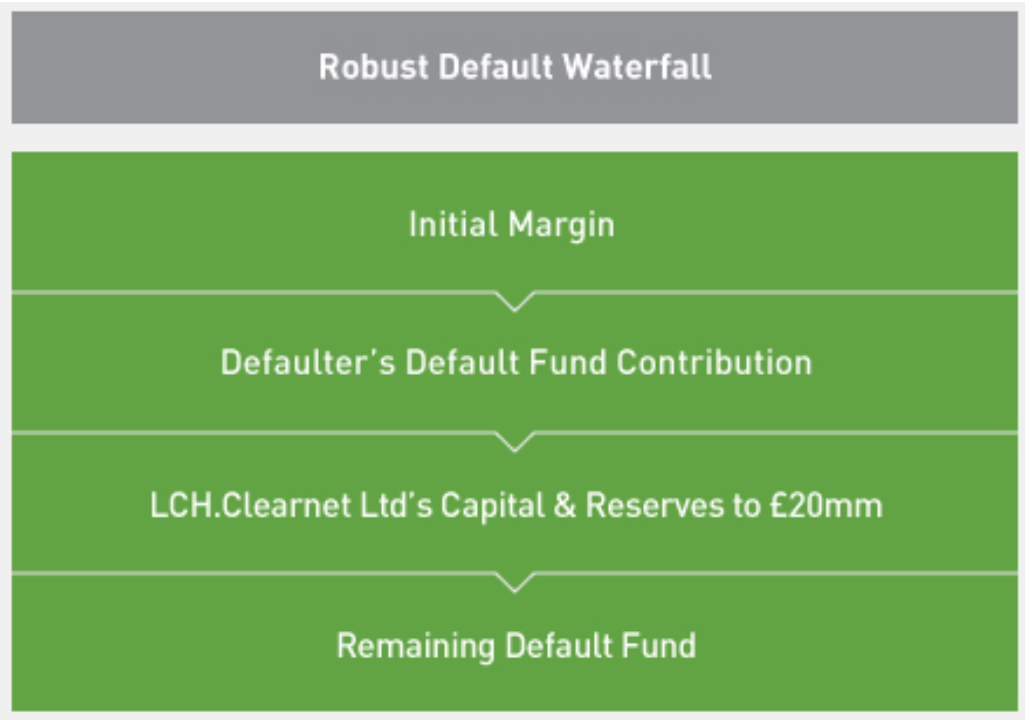

Figure 4.1

Robust default waterfall as applied by SwapClear ${ }^{1}$

discuss how we deal with the margins and default fund contribution deposited with the CCP and the principle of novation that is one of the main devices the $\mathrm{CCP}$ employs to mitigate risk.

\footnotetext{
${ }^{1}$ http://www.swapclear.com/service/risk-management.html
} 
Lastly, we expand on how a failure of the CCP is treated in our model.

\subsection{Default Fund Contribution}

GCMs are obliged to contribute to a default fund. This fund is maintained by the CCP to cover any residual losses arising from counterparty failures after claiming the collateral posted as initial and variation margins by defaulting counterparties. Such losses can arise from extreme changes in the values of the defaulted portfolios. There are various ways in determining the required contribution. This contribution is sometimes set equal to a fixed percentage of initial margins. The general approach is to base the amount required on a stress-test. An instance is where this stress-test consists of using worst case scenario's of the participant's initial margin requirements in combination with a default of the two largest members and theoretical market scenario's.

We find various definitions for how the required contribution is determined. One of the membership criteria of SwapClear" ${ }^{2}$ states that "all Clearing Members will be required to contribute, in cash, to the segregated SwapClear Default Fund, the amount of which will be based upon:

1) A minimum Default Fund contribution of $£ 10$ million per Clearing Member.

2) Risk weighted contribution calculated by taking an averaging period for initial margin".

It further states that "SwapClear Clearing Members will be obliged to provide additional unfunded Default Fund contributions limited to one unfunded assessment per member default to a maximum of three in six months." An extensive treatment of how these requirements are calculated can be found in the Default Fund Rules (March 2013) ${ }^{3}$ published by LCH.Clearnet. In line with what was mentioned earlier, "On each business day, the Clearing House will determine a "Combined Loss Value" in respect of each of the 60 preceding business days. The Combined Loss Value in respect of a particular day will be the sum of the largest and the second largest stress - testing loss incurred on that day in relation to Swap Clear Business (for a given scenario)." It further states that "SwapClear Segregated Fund Amount" is the largest of the 60 Combined Loss Values determined, as described above, plus $10 \%$. There are of course more details mentioned in the document Default Fund Rules (March 2013) to which we refer the interested reader.

The least complicated description for the required amount of the default fund contribution is a fixed percentage of initial margins. A GCM's contribution was set to $10 \%$ of initial margins or $£ 2$ million, whichever of the two is larger. As it is not our aim in this paper to overcomplicate matters we simply assume in our model that the default fund contribution equals

$$
\Theta_{\beta}=10 \% \text {. }
$$

Whether this assumption is sufficient to mitigate residual losses of the 2 biggest GCMs is a completely different issue, which deserves separate investigation and is rather irrelevant for our analysis.

\footnotetext{
${ }^{2}$ http://www. swapclear.com/service/becoming-a-member.html

${ }^{3}$ http://www.1chclearnet.com/rules_and_regulations/ltd/
} 


\subsection{Margins}

Initial margins are calculated on every cleared and every to be cleared IRS. These margins (collateral) have to be posted before an IRS is cleared with the CCP. For instance, SwapClear determines initial margins in such a way that in the event of a default of a GCM it covers the potential losses that would have occurred on the portfolio within recent history and is set at the portfolio's observed worst-case-five-day loss over the last five years. A Value at Risk (VaR) model is applied to calculate the initial margins. This is a historical simulation using a 5 year history of market scenarios and a $100 \%$ confidence level. To allow hedging of a defaulter's portfolio a 5 day holding period is applied. Losses that may have occurred on the portfolio during market events occuring more than five years ago are stress tested against the default fund.

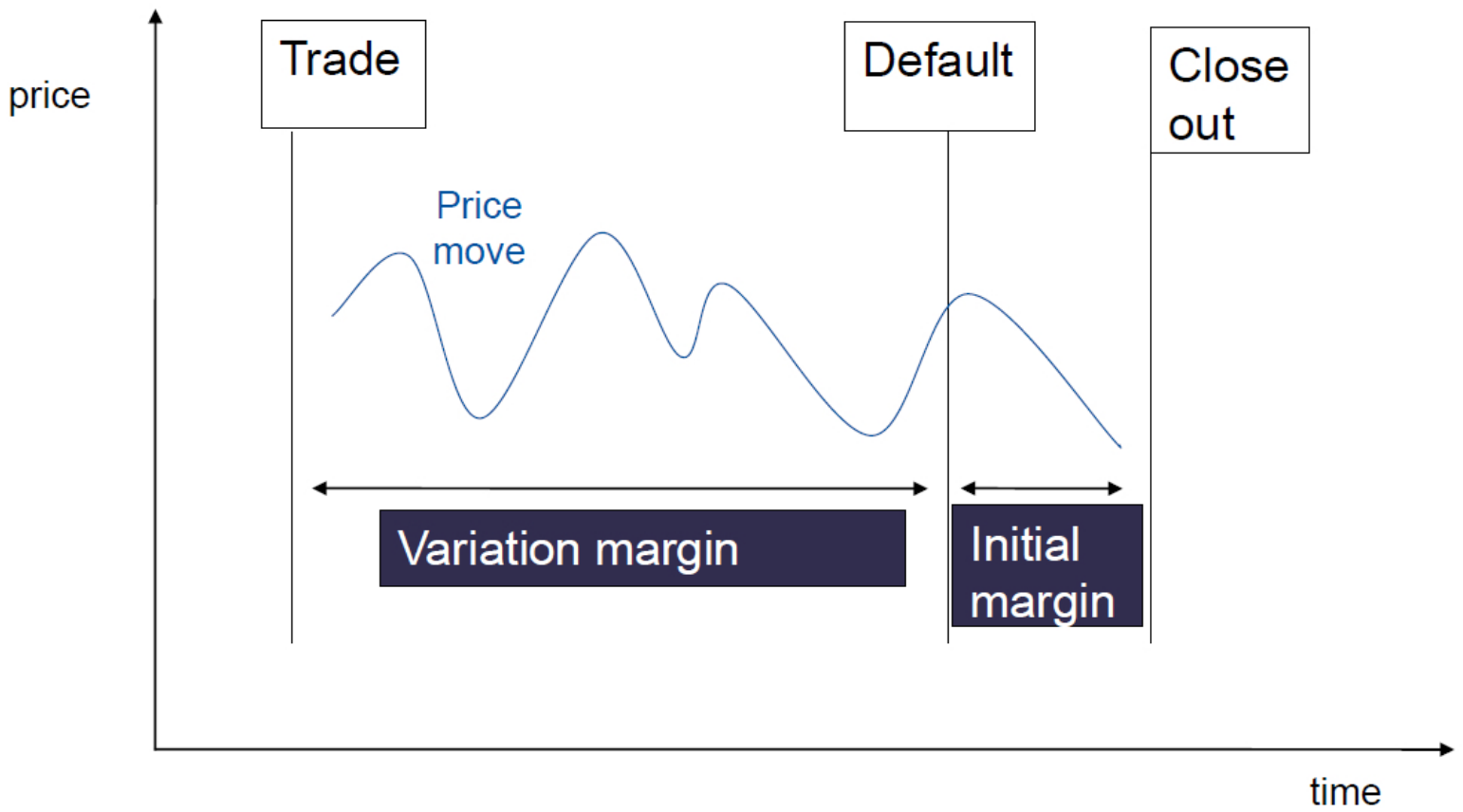

Figure 4.2

(Source: lchclearnet.com) An example showing the difference between the purpose of the variation margins and the initial margins. Variation margins are closely related to the price movements under normal trade and market conditions. Initial margins are specifically meant to cover any possible losses the CCP might face in case of a default under normal market conditions. ${ }^{4}$

Figure 4.2 shows how initial margins are applied to cover adverse price movements that occur after the default of a GCM. The variation margins cover any adverse price movement prior to the default unlike the initial margins. Hence, the initial margins cover any potential losses the CCP might face in a circumstance where it is faced with closing out or transferring the defaulter's portfolio within a certain (short) time period. Similarly, only normal market conditions are considered for determining the required initial margins since the default fund is designed to cover stressed market conditions. As our model does not include these effects we opt for a simple representation of $\Theta_{\alpha}$. We assume that the CCP requests initial margins from all GCMs

\footnotetext{
${ }^{4}$ http://www.Ichclearnet.com/images/lch\%20clearnet $\% 201$ td $\% 20-\% 20$ initial $\% 20$ margin $\% 202012$ _tcm6-44535.pdf
} 
equal to $10 \%$ of the notional principle value of a swap contract. Thus, each GCM is obliged to post collateral equal to $10 \%$ of the total notional principle value of its portfolio as initial margins.

The variation margins are directly computed from the price movements, which are directly calculated within the model at each time point $t \geq 0$. Since this is the case, we need no extra assumptions to determine these values.

\subsection{Novation and Netting}

Once two parties have agreed to a trade, which happens directly between banks or via brokers, these matched trades reach the CCP. The CCP subsequently takes the responsibility of clearing these trades. The original trades are under bilateral agreements for the two parties, e.g. their International Swaps and Derivatives Association (ISDA) agreement. The CCP replaces the original contracts between the original counterparties with two new contracts; one between the buyer's GCM and the CCP, and another between the CCP and the seller's GCM. By stepping into the trade, the CCP effectively becomes the only legal entity that the GCMs need to be concerned with as they do not have any exposure to their original counterparty any more. Figure 4.3 shows an example of how this works. In a bilateral system all parties are exposed to one another. In contrast, The CCP is exposed to all parties, while each party does not have any exposure any more to the original counterparties, but only to the CCP.

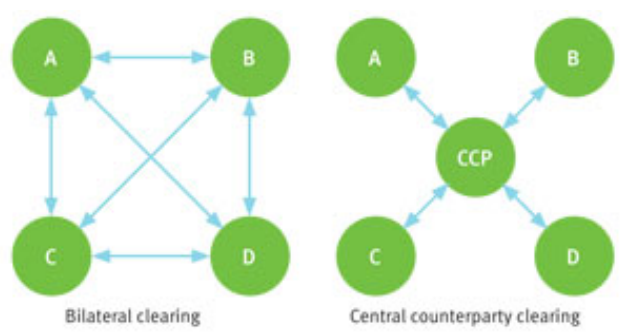

Figure 4.3

Two clearing systems are shown. The left one is a bilateral clearing system while the right one clearing system via CCP.

As novation occurs at a large scale, trades are netted. Thereby reducing the number of open positions and increasing capital efficiency. To see how this works we look at the example given in figure 4.4. Consider an economy with three parties, GCM 1,2 and 3 and one security traded, say A. GCM 1 has agreed to two trades; one with GCM 2 and one with GCM 3. GCM 1 sells security A to GCM 2 and agrees to the same trade between GCM 3 with an opposite position. The novated trades are shown in the figure 4.4. It is obvious that the CCP has no exposure to GCM 1 when all its trades are netted as shown in situation B in figure 4.4. CCPs greatly reduce operational risks, operations costs and financing costs through netting. Not only is this effect maximized when novation occurs at large scale, but also when trades are more standardized, for obvious reasons.

This example might not translate directly to an implementation in our simulation exercise, since we assumed that GCMs do not hold both legs of the same IRS. It is however of interest 


\section{Situation A}

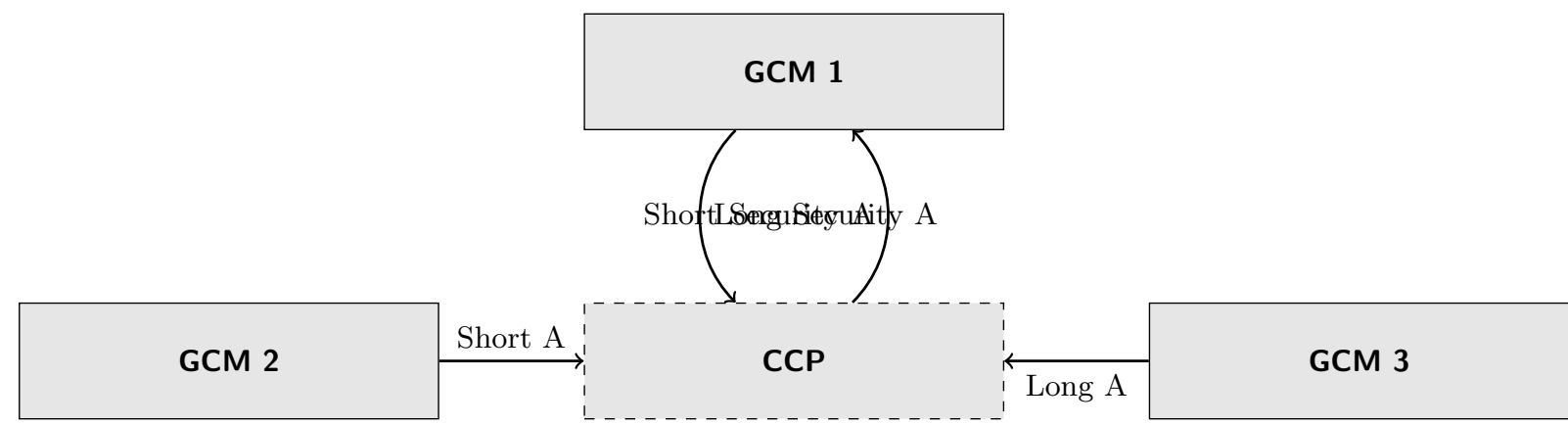

Situation B

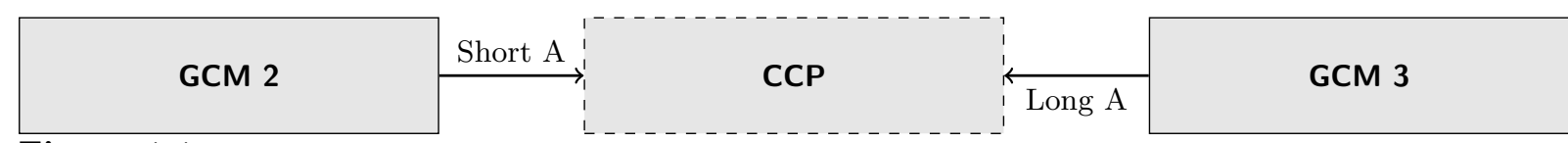

Figure 4.4

Situation A shows the novated trades before netting. Situation B shows the exposures when obligations are netted

to consider the situation where the CCP might have to transfer the portfolios of more than one defaulter. As the transactions of each GCM can not be set off against each other at market values by the CCP since it still has to fulfil its obligations with the non defaulting counterparties. It is however possible to set off any contracts against each other in case of more than one default without the need to transfer these contract (or portfolios). This would be the case in the example given in figure 4.4 if both GCM 2 and GCM 3 would default at the same time. Even in light of the fact that these parties were not each others counterparties, the CCP would still be able to net these transactions. This method can limit the exposure of the CCP when more than one default occurs at the same time and somewhat relieve the financial system of any residual losses resulting from the transfer of the defaulter's portfolios. We apply a similar approach in our simulation exercise where contracts are set off against each other when more than one default is registered whenever possible.

\subsection{Omnibus Account}

CCPs vary considerably in the way the segregation of assets (collateral) is handled. The segregation model employed by the CCP strongly affects the risk associated with a default faced by the non defaulters. Depending on the segregation model a GCM (or its client) can be exposed to a GCM, a GCM and other clients, the CCP or any combination of the above.

Some CCPs keep GCM and their customers assets in segregated accounts, but all customer assets are all held in an omnibus account. If a default of a customer (GCM functions as broker for its customers) leaves a deficit in its account balance owed the CCP, while the GCM has insufficient capital to cover the deficit, non-defaulting customers are at risk to the GCM and 
hence the CCP to utilize their assets in the omnibus account to honour the monies owed by the customers of other GCMs. A more extreme case would be a segregation model where all assets are held in an omnibus account. This means that all GCMs are at risk of having the CCP utilize the monies in their accounts to cover for any residual losses of a defaulting GCM (or customer). Since we only consider GCMs in our model it is of course trivial to opt for the second model.

\subsection{CCP Failure}

Historically, CCPs have defaulted. One of the first, albeit not exactly a CCP, was the New York Gold Exchange Bank. Two defaults of large gold speculators lead to the failure of the New York Exchange Bank. A more recent case, was the Casisse de Liquidation failure in 1974 and the Kuala Lampur Commodity Clearinghouse failure in 1893. The 1987 stock market crash, referred to as the "black Monday", led to the failure of the Hong Kong Futures Excahnge Clearing Corporation.

The CCP could default on its obligation if all its resources are exhausted (Margins, Default Fund and its own capital) as a result of GCMs failing to perform on their contractual obligations. CCPs are designed to reduce system risk but in reality, CCPs may become a node of concentration. This would make the CCP the most basic case of 'too big to fail'.

Even if the chances of a failure of a $\mathrm{CCP}$ are remote at best, several resolution mechanism have been proposed to address such a failure if one is to arise. Making the defaulted CCP's contracts bilateral is one of the proposed policy responses. This should be possible since a CCP has a zero net position in every trade it clears. As mentioned earlier, for each contract there is both a seller and a buyer. As each trade has been agreed on before it is novated, this means that each buyer and seller of each contract with an open position with the CCP could be matched exactly by creating bilateral contracts between the two parties. We opt in our simulation exercise for this approach. Creating bilateral contracts between the two parties takes us back to a bilateral OTC market. This enables us to take any residual losses after the failure of the CCP into account. It is quite possible that the failure of a CCP leaves the GCMs in a very vulnerable position, especially when all assets have been used up by the CCP while it was still operating. Wrong way risk might play a considerable role in such a case too. 


\section{Defaults and Loss Contagion}

Our simulation experiments are constructed to investigate how shocks affect the financial system. Our starting point is thus a first default, i.e. a fundamental default, that acts as a cause of all possible contagion losses and contagion defaults. The way we set up the system allows for more than one fundamental default. This follows from the fact that we simulate a stochastic path for the value of each contract until a loss big enough leads to at least one default. In other words, it is quite possible that a more than one GCM suffers enough losses on a certain contract leading to two or more simultaneous defaults. The losses in both systems, a system with central clearing and one without, can be compared by taking the same default event as a starting point for all contagion losses and contagion defaults in both systems. This enables us to compare the results obtained for both systems.

We need to make a few remarks about our approach in simulating defaults and the way the $\mathrm{CCP}$ reacts to these losses. We treated in the previous section a few "defence mechanisms" the CCP employs to mitigate default risk and to cover any residual losses the non-defaulting GCMs may face. The methods take into consideration both the market conditions and the financial health of each individual GCM. CCPs would most likely take sufficient measures when extreme market conditions arise or extreme losses are faced by GCMs. A practical example would be the default of one of the biggest players on the OTC derivatives market, Lehman Brother, in September 2008 during the financial meltdown of 2007-2008. LHC.Clearnet was able to successfully manage Lehman Brother's $\$ 9$ trillion (Gross value) IRS default within the margins held by the CCP. We stress that we do not take these effect into consideration in our model for the sake of simplicity. As mentioned in the previous section, for each component of the protection mechanisms employed by CCP, we opted for a simple implementation. Thus, our model simply compares the spread of contagion in a centralized system compared to a bilateral one in a world where the transition form normal market conditions to extreme market conditions where extreme losses are incurred by GCMs is almost instantaneously. We keep silent on how remote the probability is of this happening and simple compute the losses in our system in such a setting.

As mentioned above, the fundamental default(s) is (are) the starting point of the any possible cascading losses. We briefly discuss the way we determine these defaults. Next we make the distinction in how losses are spread within both system, a system with central clearing and a system with bilateral trades. we first discuss how we treat losses in a system with central clearing and how losses spread in this system. We then discuss how we handle a possible CCP failure in our model. Lastly, we briefly discuss the spread of losses and contagion defaults in a system with bilateral trades which completes the default dynamics in our model.

\subsection{Fundamental Defaults}

We mentioned in section 3.2.1 that each of the $M$ IRSs are modelled by its own Wiener process $W_{t, m}$. By simulating this process for each IRS and summing over the values of all contracts in the portfolio held be a GCM we compute the portfolio value $\Delta_{i} \in \mathbb{R}$. Figure 5.1 
shows an example of a simulated path of an arbitrary IRS $m$ with a $\hat{\sigma}_{m}$ of our choosing.

Figure 5.1

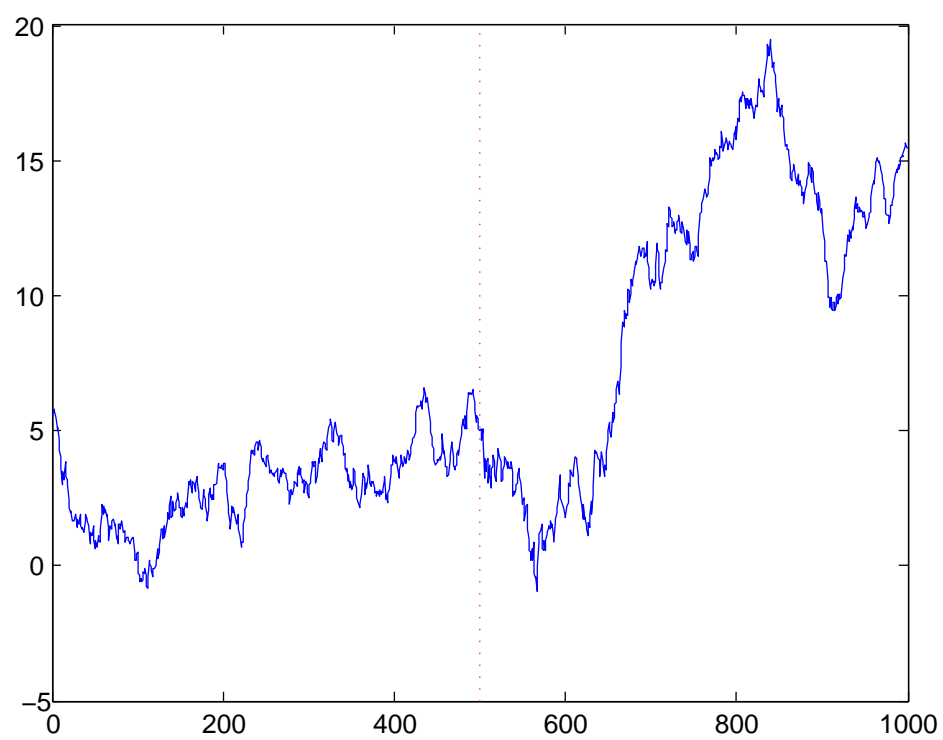

An instance of a simulated path $V_{t, f i x, m}$ for a a given $\hat{\sigma}_{m}$. The value of the IRS is determined for each $0 \geq t \geq 1000$. At $t=500$ we inflate $\hat{\sigma}_{m}$ to obtain a more volatile series.

The default fund contribution and initial margins are determined based on the portfolio and not based on its value. The variation margins, $\varepsilon_{i, t}\left(V_{i, t}\right)$, are however determined from the total value of the portfolio. We do not need to consider each individual IRS. This means CCPs require variation margins from each GCM with respect to its total position, $\Delta_{i}<0$ for $i=1, \ldots, n$. A GCM defaults if

$$
\Theta_{\alpha} \Delta_{i}+\Theta_{\beta} \Theta_{\alpha} \Delta_{i}+\varepsilon_{i, t}>c_{i}
$$

and thus being unable to post collateral to cover its position. We simply simulate the values $V_{i, t}$ for $i=1, \ldots, n$ and $t>0$ until a first, or more, default(s). This approach allows us to determine the exact exposure and posted collateral since these are computed for each GCM at each time step. It is also possible to aggravate the losses incurred by GCMs by blowing up $V_{i, t}$, by introducing extremer adverse price movements. We will especially look at the effect of different shock sizes in our simulation experiments for small-world networks where the shock size appears to have a non-linear effect on contagion.

\subsection{Central Clearing and Loss Contagion}

A fundamental default, or more than one, results in a loss equal to $h_{i}$ or $\sum_{i \in \Omega_{u}} h_{i}$, with $\Omega_{u}$ the set of contagion defaults in the $u$ th round. This loss is first mitigated by the variation margins and initial margins of the defaulted GCMs. If some residual losses persist, the initial margins of the non-defaulting GCM are utilized to cover for these losses. This is the case since we assumed that all assets are held in an omnibus account. Finally, the collateral in the default fund can be 
utilized if not all losses are covered for by the initial and variation margins.

IRS contracts of the defaulted parties are set off against each other if possible. Since the CCP always maintains a zero net exposure to the GCMs. The CCP achieves this by transferring the contracts to financially healthy GCMs. In our simulation exercise we assume that all non-defaulted parties are financially healthy, which is of course not always the case. It is quite possible that an operating GCM is in danger of default. In our model the CCP demands after the transfer of the IRSs a contribution as discussed earlier.

After the CCP has transferred all the IRSs of the defaulted parties, we compute the extra collateral each GCM has to post in the second round. This can either be an extra contribution to the default fund if this is eroded in the previous round or a simple margin call for all the new contracts held by a specific GCM. If any GCM is unable to perform on its obligations, it defaults, and the process is repeated. We stop this recursion when no contagion defaults are found in a round.

\subsubsection{CCP Failure and Transition to a Bilateral System}

In view of the fact that the $\mathrm{CCP}$ can not refund the default fund more than ones, it is of course possible that the $\mathrm{CCP}$ fails at a certain point. As mentioned earlier we simple carry out a transition to a bilateral OTC market. GCMs become each other counterparties instead of the CCP. All collateral of the GCMs held by the CCP is lost in this case and all further computations are handled in line with our approach below.

\subsection{Bilateral OTC Market and Loss Contagion}

The losses in a bilateral OTC market are more straightforward compared to a system with central clearing. Financial institutions now have to deal with counterparty credit risk. Counterparty credit risk is the risk that a counterparty to an IRS will default prior to the expiration of the contract. Thus not making all the payments required.

There are two cases we distinguish when a counterparty defaults as Pykhtin and Zhu (2007) propose. The first case is were the contract value is positive for the party, say pension fund, at the time of default. This party closes out its position by paying the defaulting counterparty the market value of the contract and enters into a similar contract with another party and receives the market value of the contract. The net loss in this case equals zero. The second case is when the contract value is positive for the party. It now has to close its position while receiving nothing in return from the defaulting counterparty. It enters into a similar contract with another paying the market value. The loss now equals the contract's market value. This means that the total loss incurred by party $i$ equals

$$
s_{i}=E[L o s s]=\sum_{j \in \Omega_{u}} \sum_{i} \max \left\{V_{i, j}, 0\right\} .
$$


Equation 5.2 states that the total loss party $i$ incurs equals the sum of all contracts with positive values with a defaulting counterparty $j \in \Omega_{u}$, with $\Omega_{u}$ the set of defaulting counterparties. This enables us to compute all losses incurred by the counterparties when defaults happen. Any margins held by party $i$ before the default of counterparty $j \in \Omega_{u}$ is subtracted from the aggregated losses incurred by party $i$ given by equation 5.2 . If

$$
q_{i}=\sum_{j \in \Omega_{u}} q_{i, j}
$$

is the total collateral held by party $i$, then the losses are given by

$$
h_{i}=\left|\max \left\{0, s_{i}-q_{i}\right\}\right| .
$$

The shock $h_{i}$ is absorbed by the capital $c_{i}$ of party $i$. If $h_{i}>c_{i}$, then the party defaults, otherwise it withstands the shock. At each round we calculate all losses suffered by each party and how many parties default. If new defaults happen we repeat the process above, otherwise we obtain the total number of defaults and stop the simulation. 


\section{Simulation Results}

Recall that a range of networks were considered for modelling the financial network. We considered a set of parameters to model our network and GCMs which were described by their balance sheets. Our results are mainly of comparative nature. We compare the results obtained for the same network with central clearing and with bilateral trades. Specific parameters are varied in our simulation experiment to see how the results compare over a range of values. To be more precise, we look specifically at how the resilience of both systems compares when we vary the capitalization of GCMs. We further examine how concentration affects the capability of shock absorption within the system. By concentration we mean the number of market participants $n$ in the network.

The first step in the simulation experiments is to generate a random graph. The second step is to construct all the balance sheets and portfolios of the GCMs and CCP. Portfolio values are simulated thereafter till we obtain a fundamental default. Next, the spread of the shock through the system and contagion are calculated from which we obtain the overall number of defaults and the total losses to the system. This Monte Carlo simulation experiment is conducted by repeating these steps for 10.000 times. The analysis is subsequently based on the averages obtained from these simulations.

\subsection{Random Networks}

The effect of the system size in terms of number of participants, i.e. concentration, on the rate of default and the relative losses in the system is first investigated. The results for both systems, with bilateral and central clearing, are compared. Figure 6.1(a) compares the obtained default rates, given the number of participants $n$, for both systems. Figure 6.1(b) shows the average capital loss given the number of participants $n$. The red line represents the results for a system with bilateral clearing while the blue line represents the results of a system with a CCP. The dotted lines show the range were $95 \%$ of the observations lie. We let $n$ grow until the graphs stabilize, which is around $n=80$ in this case.

Figure 6.1(a) shows the average default rates. The bilateral system does not appear to benefit from an increasing number of market participants unlike a system with a CCP where the diversification effect is apparent. The model for the OTC market was build based on the assumption that the total foreign assets are kept fixed. This means that for more concentrated markets, the size of each GCM is relatively smaller compared to the total size of the market. The CCP benefits from this fact in two ways. First the incurred losses on average are smaller for a larger system and the CCP can relatively utilize more assets to cover for residual losses. This benefits do not appear in system with bilateral clearing. Defaulters transmit losses directly to their counterparties in contrast to a system with CCPs.

Figure 6.1(b) shows the total capital losses in both systems. We first note that there appears to be no linear relation between the default rates and average capital losses. The benefit from an 


\section{Figure 6.1}

Defaults as a function of $n$

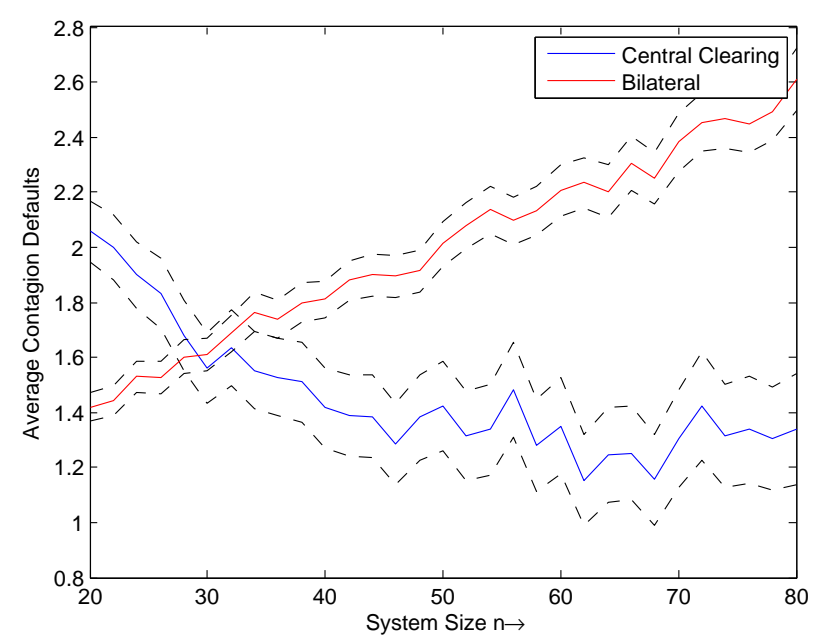

(a) Default rates

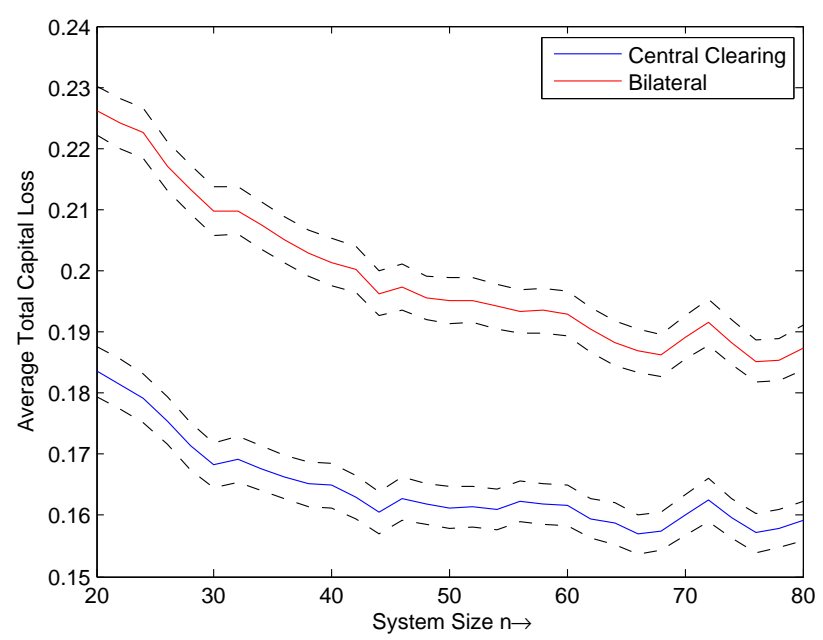

(b) Average Total Capital Loss

increase in market participants in both systems is however evident. Furthermore, there is once again a significant advantage to central clearing of OTC derivatives. A consistent decrease in losses when IRSs are cleared with a CCP is substantial. The system appears to be consistently more resilient. Increased diversification benefits appear to get smaller for systems with more market participants.

Figure 6.2 shows the probability of failure of the CCP for different concentration levels. We find that the CCP benefits in a similar fashion as all the market participants when more GCMs are present. The reason for this effect seems to be the same as mentioned above. The size of the shocks for a system with more participants is relatively smaller and easily mitigated by the CCP because of the relative higher capability of raising more funds to cover for residual losses.

Next, the resilience of the financial system given the capitalization of GCMs is investigated. Figure 6.3(a) shows the default rates and figure 6.3(b) shows the capital losses. As expected, a significant decrease in both default rates and capital losses is the result of larger buffer. GCMs are capable of absorbing losses much better when more capital is kept as a buffer against shocks. We find that the benefit of central clearing of IRSs appears to vanish when GCMs decide to keep more capital to absorb any losses arising from the default of a counterparty.

A slightly lower average default rate is found when IRSs are cleared with CCP for $5 \% \leq$ $c \leq 20 \%$. A similar pattern is observed for the average capital losses in figure 6.3(b). There appears to be no benefit to central clearing of OTC derivatives for $c>0.2$, which is extremely high compared to a more practical range of $0.05 \leq c \leq 0.08$, where central clearing shows some, perhaps small, benefits.

Figure 6.4 shows the probability of failure of the CCP given the buffer of the GCMs. The results are in line with the average default rates obtained in this simulation experiment. We noted that GCMs are able to withstands shocks much better given a higher capital buffer. This 
Figure 6.2

Probability of CCP Failure

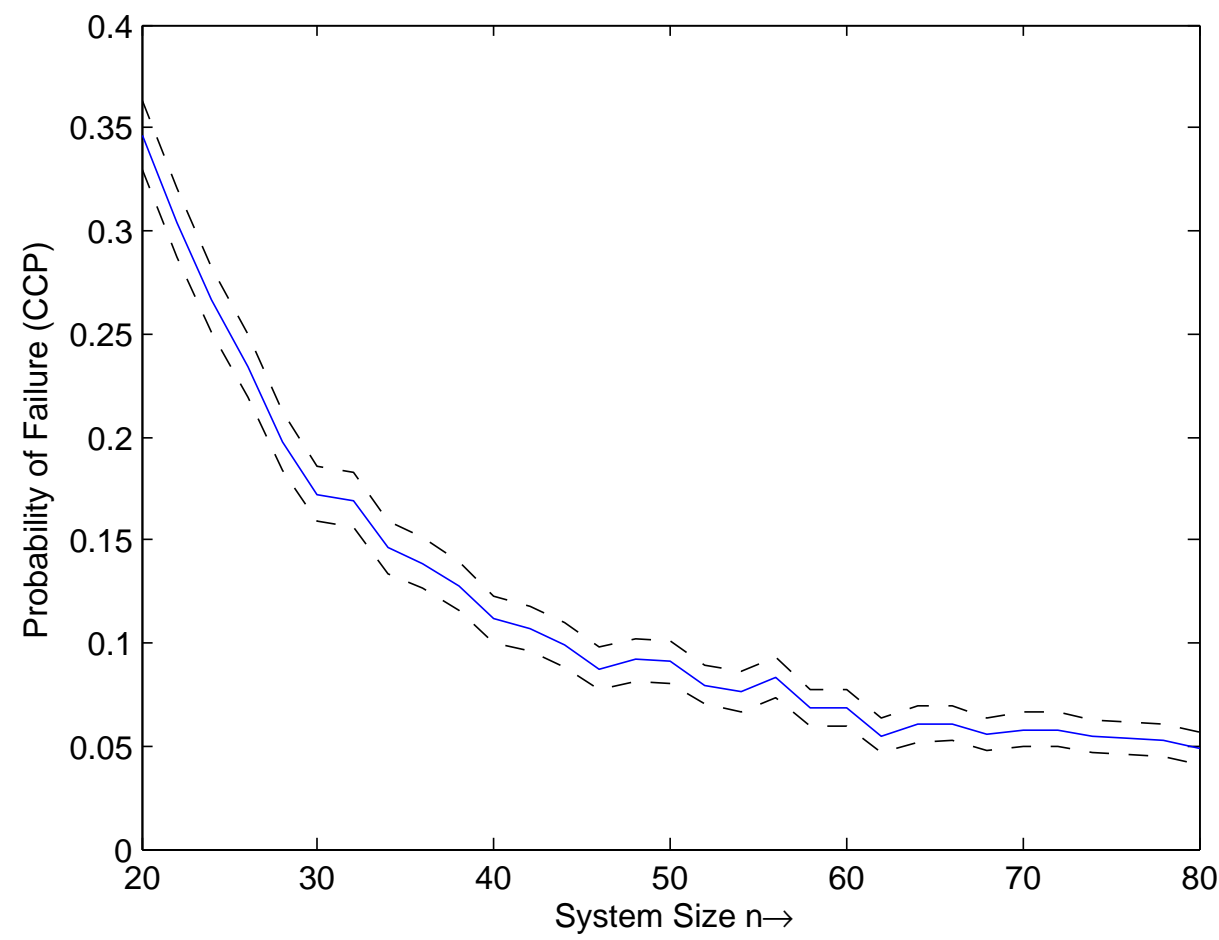

obvious conclusion translates to a much smaller chance of the CCP getting into trouble for two reasons. GCMS can much better mitigating any residual losses the CCP might have to cover when unexpected losses are incurred, causing GCMs to less likely default as a result of contagion because of the higher capital buffer. This leads to smaller of not any contagion defaults the CCP has the handle.

\section{Figure 6.3}

Defaults as a function of $c$

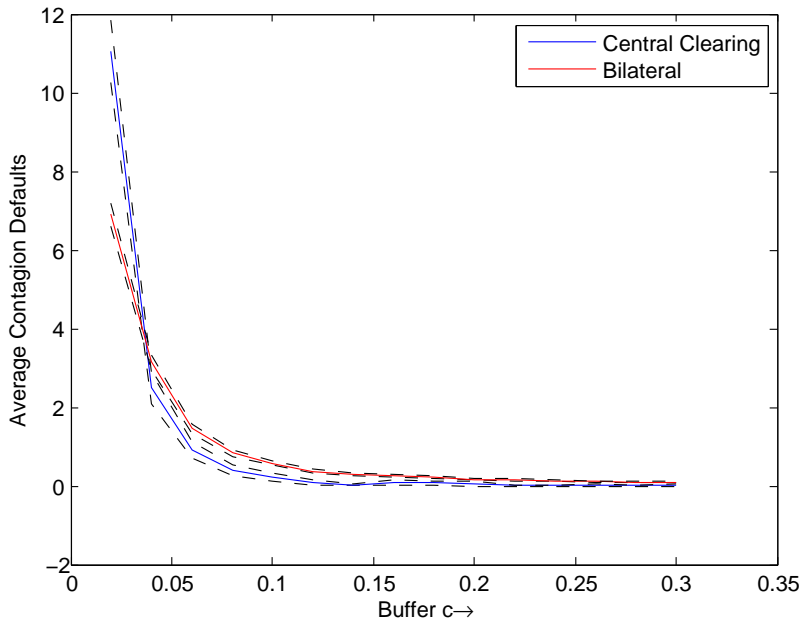

(a) Default rates

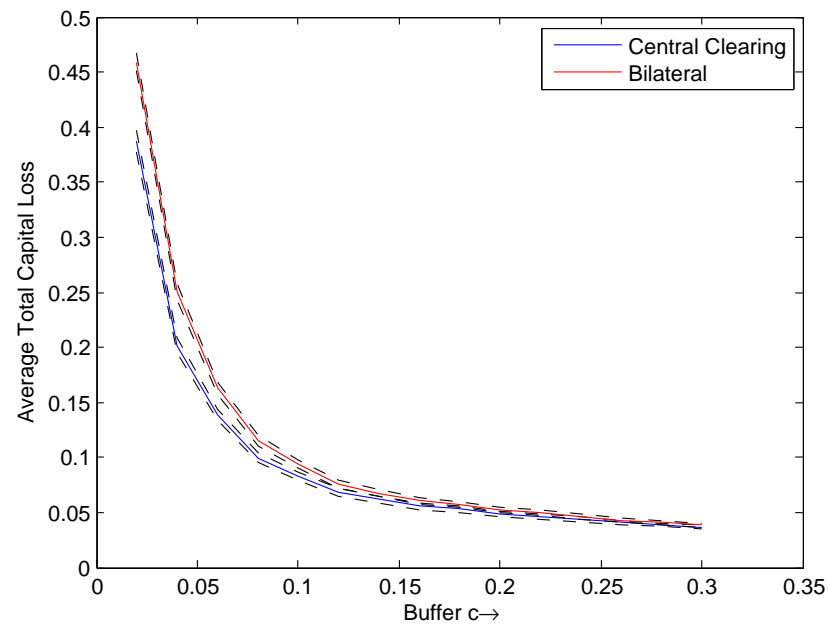

(b) Average Total Capital Loss 
Figure 6.4

Probability of CCP Failure

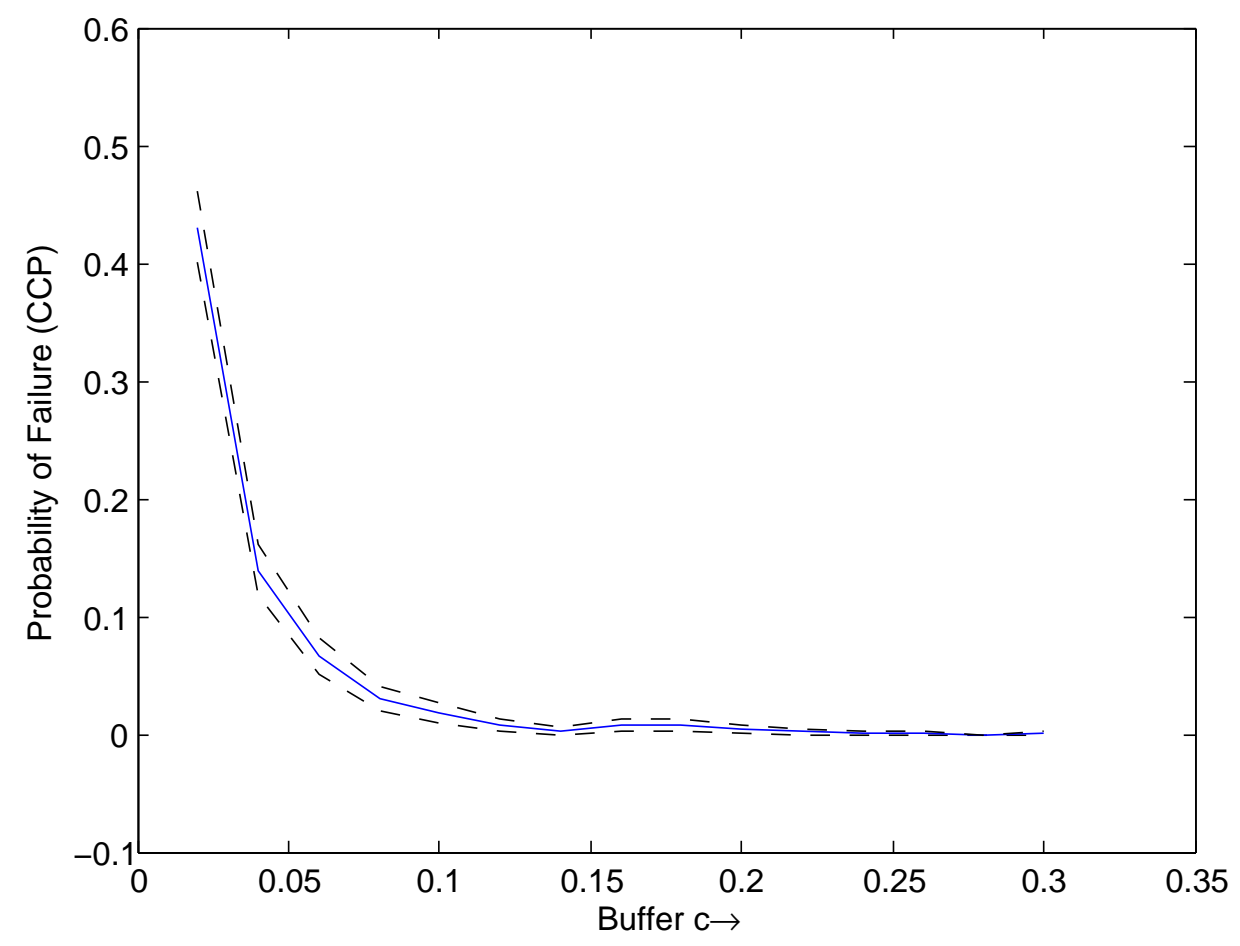

\subsection{Random Tiered Networks}

\subsubsection{Concentration and contagion}

Simulation similar to the ones given in the previous section are repeated for random tiered structured networks. Random tiered networks consist of two classes of GCMs, large and small GCMs, unlike the random networks where all GCMs are ex ante of similar size. Figure 6.5(a) and figure 6.5(b) show the average default rates and average capital losses, respectively. Similar patterns are observed, like the ones obtained for the random network, for the average default rates and average capital losses. However, the default rates are slightly lower for the random tiered networks compared to the random networks. The default rates now decrease from an average of 1.9 contagion defaults in a system with $20 \mathrm{GCMs}$ to an average of 0.4 for a system with 60 GCMs. In contrast, we obtained in figure 6.1(a) a default rate of 2 for a system with $n=20 \mathrm{GCMs}$, stabilizing at an average default rate of 1.2 for a system with $n>60$ GCMs. However, we do not observe this significant decline in default rates for a bilateral system. The average capital losses appears to lie somewhat at a lower level compared to the results for random network.

We suspect that this effect is explained by the fact that a default of a small GCM in a random tiered network is insignificant. This might lead to a smaller average default rate. This hypothesis is investigated by only considering the average default rate when a large GCM defaults. Our suspicion is that the average default rate will be considerably larger in this case. Figure 6.6(a) and figure 6.6(b) show the average default rates and total losses from contagion, respectively. As we suspected, the default rates show that there is a much higher contagion risk when a 


\section{Figure 6.5}

Defaults as a function of $n$

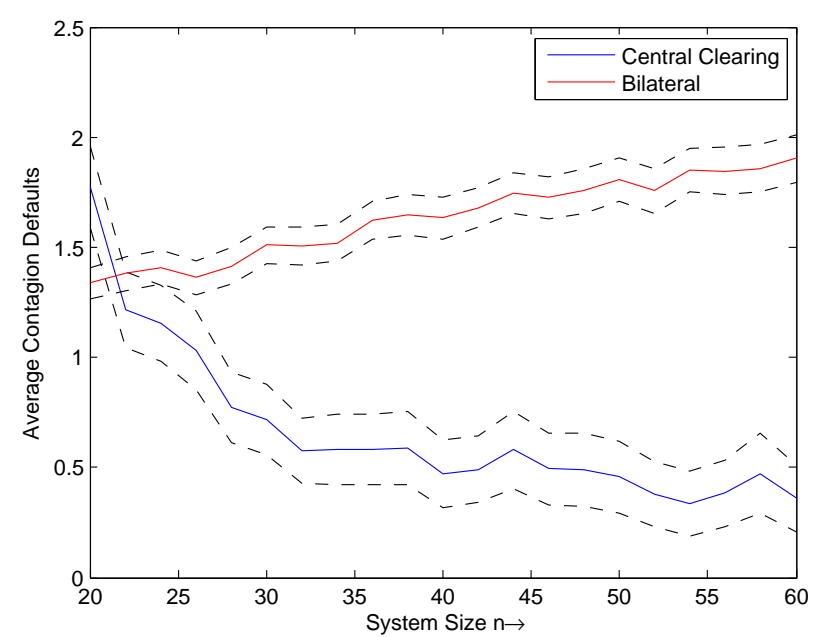

(a) Default rates

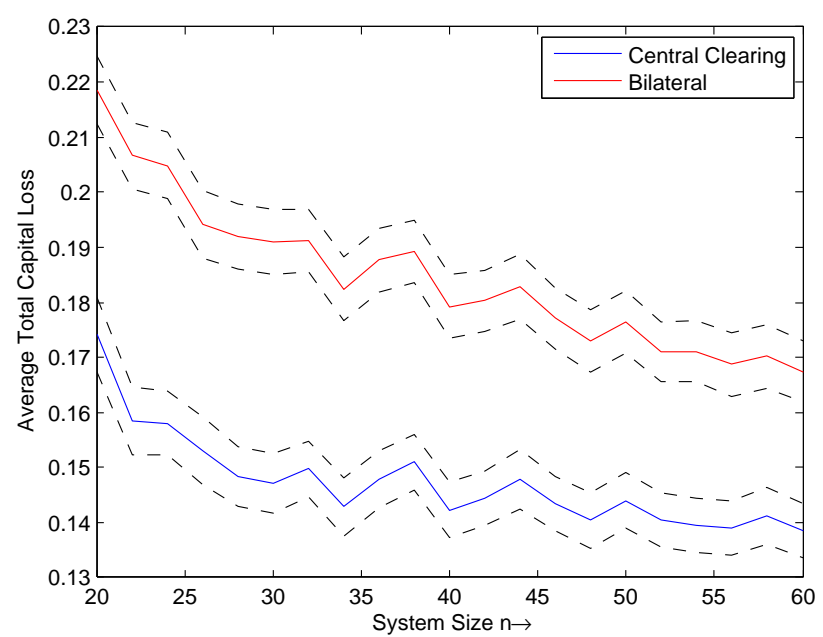

(b) Average Total Capital Loss

large GCM defaults. It is however quite interesting to see that the default rates when OTC derivatives are centrally cleared are much higher which goes against our intuition. Nonetheless, the losses in contrast are higher when a large GCM defaults compared to the results obtained before, but still smaller when OTC derivatives are centrally cleared. Considering a system with bilateral trades, losses are spread directly to the counterparties of the defaulter unlike a system with a CCP where defaults and their residual losses are covered for by all market participants. This latter leads on the one hand to a better management of the total losses suffered in the system, but can put the smaller GCMs into financial distress much faster. This explains the higher contagion defaults, but lower contagion losses on average. Cascades from failure of a small GCM are highly unlikely, which dampens the default rates considerably, thus leading to the results in figure 6.5.

\section{Figure 6.6}

Defaults as a function of $n$

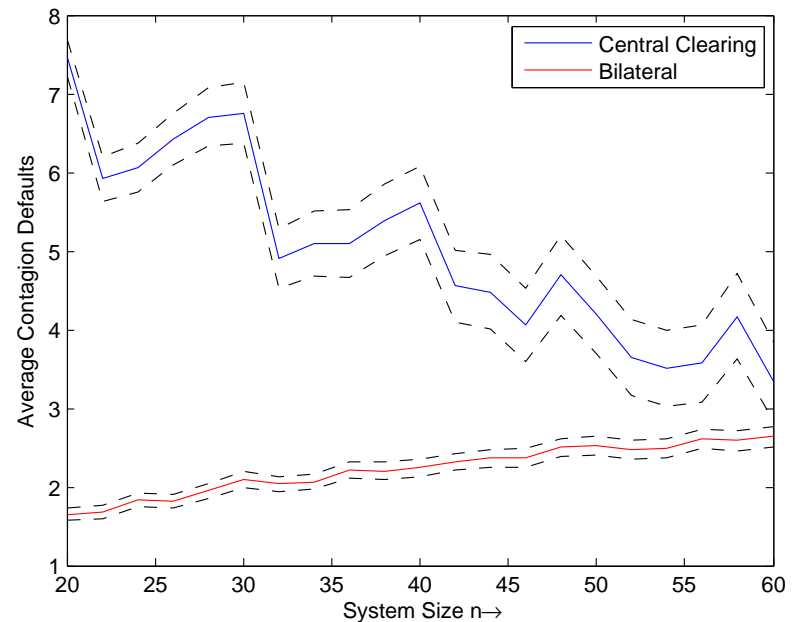

(a) Default rates

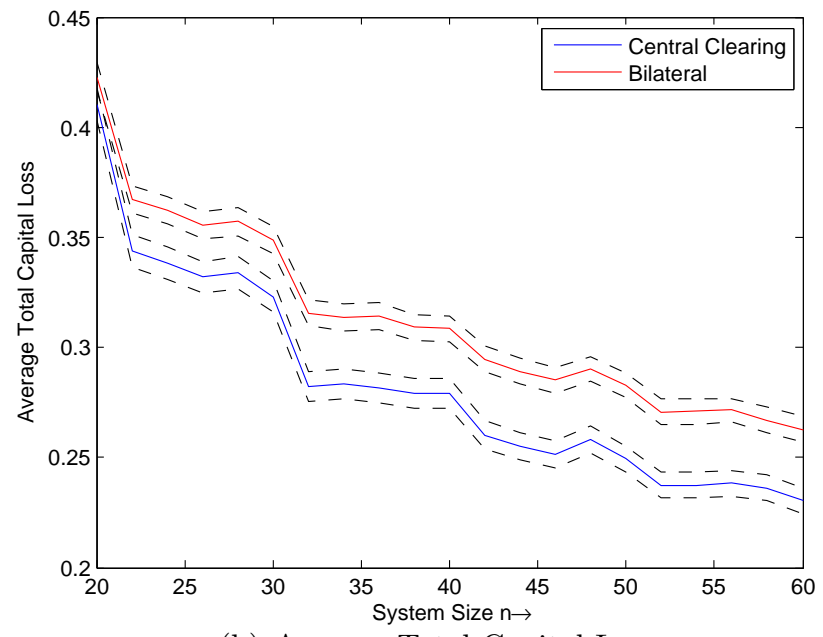

(b) Average Total Capital Loss 
Figure 6.7 shows the average probability of failure of the CCP in both settings, one where all defaults are considered as the start of the contagion cascade and one where the cascades are the result of the failure of a large GCM. The CCP benefits as we have seen earlier from a larger number of participating GCMs. More GCMs lead to larger base to mitigate residual losses as result of a stronger diversification effect.

\section{Figure 6.7}

Probability of CCP Failure

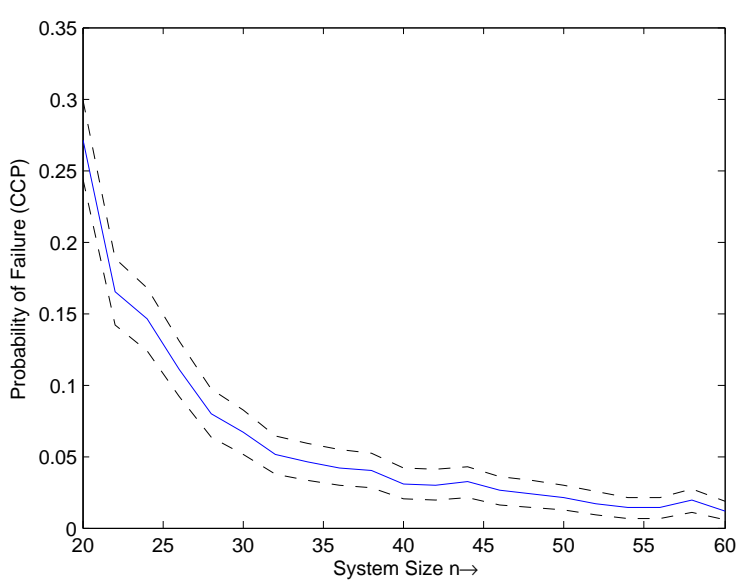

(a) CCP failure as a result from cascades from the failure of an arbitrary GCM

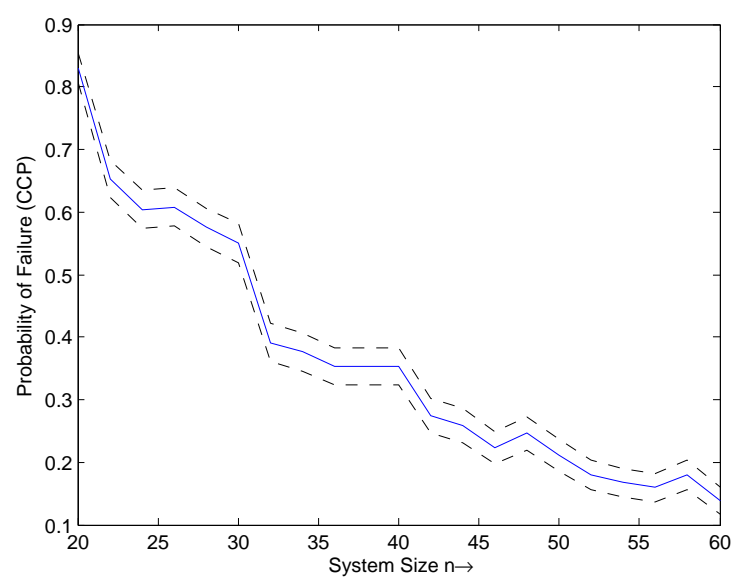

(b) CCP failure as a result from cascades from failure of an arbitrary large GCM

\subsubsection{Capitalization and contagion}

We compare the effect of a higher capital buffer on contagion defaults and losses in figure 6.8 and 6.9. The results of cascades from the failure of a random GCM are given in figure 6.8 while the results of cascades from the failure of only large GCMs are given in figure 6.9. The results shown in figure 6.8 are similar to the ones shown in figure 6.3. Central clearing of OTC derivatives appears to have a somewhat more positive effect on the default rates on the range $0.04 \leq c \leq 0.1$. The default rates of counterparties seem to be slightly higher on the same interval when bilateral trades are considered for a tiered structured network. The CCP appears to add some stability to the financial system in terms of default rates, but this does not appear to translate to a lower average contagion loss when figure 6.8(b) and figure 6.3(b) are compared.

The results of the experiment with cascades from the failure of a large GCM, as given in figure 6.9, show a surprising result. Central clearing of OTC derivatives appears to keep contagion losses limited compared to a setting where OTC derivatives are bilaterally traded. Notwithstanding this fact, the default rates do not appear to declining when a higher capital buffer is kept by GCMs. Apparently, two factors lead to these results. Recall that we set up our experiment in such a way that an event of a fundamental default would only transpire if a counterparty $(\mathrm{GCM})$ fails to perform on its obligations. This default only occurs when the capital buffer becomes insufficient to absorb the losses on a portfolio. Adding to this that large counterparties (GCMs) will only default when extreme losses accrue. Secondly, as observed 


\section{Figure 6.8}

Defaults as a function of $c$

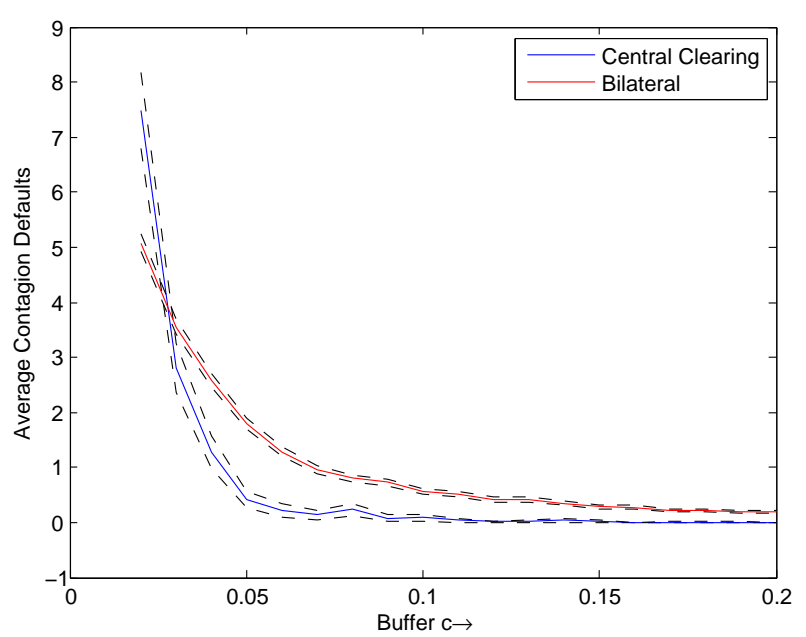

(a) Default rates

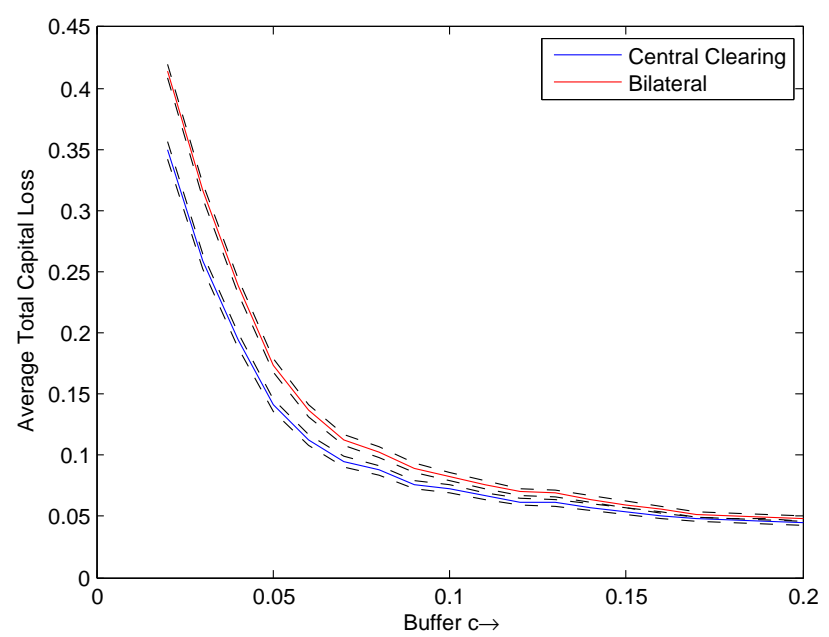

(b) Average Total Capital Loss

earlier, smaller GCMs tend to experience greater duress when derivatives are centrally cleared as a result of risk sharing. The bigger shock and additional vulnerability of the small GCMs leads to the much higher default rates as confirmed by this simulation experiment.

\section{Figure 6.9}

Defaults as a function of $c$

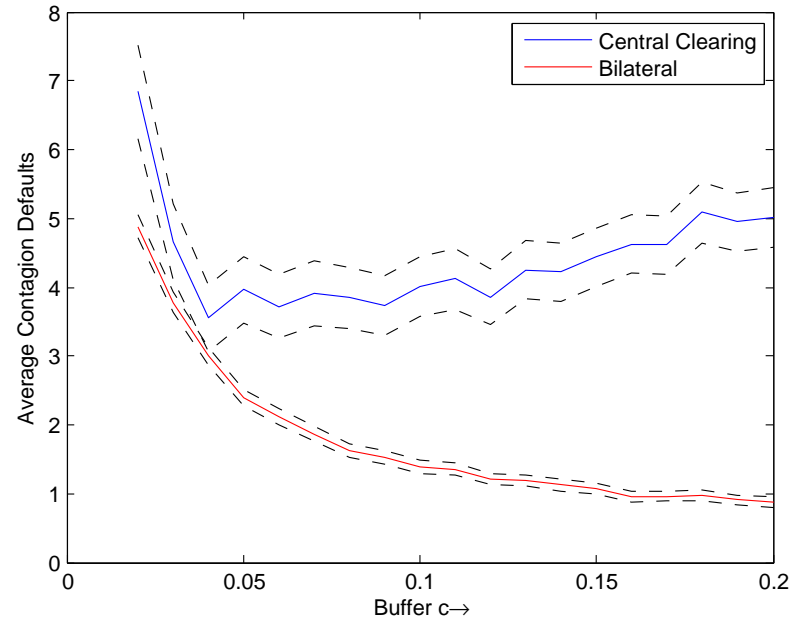

(a) Default rates

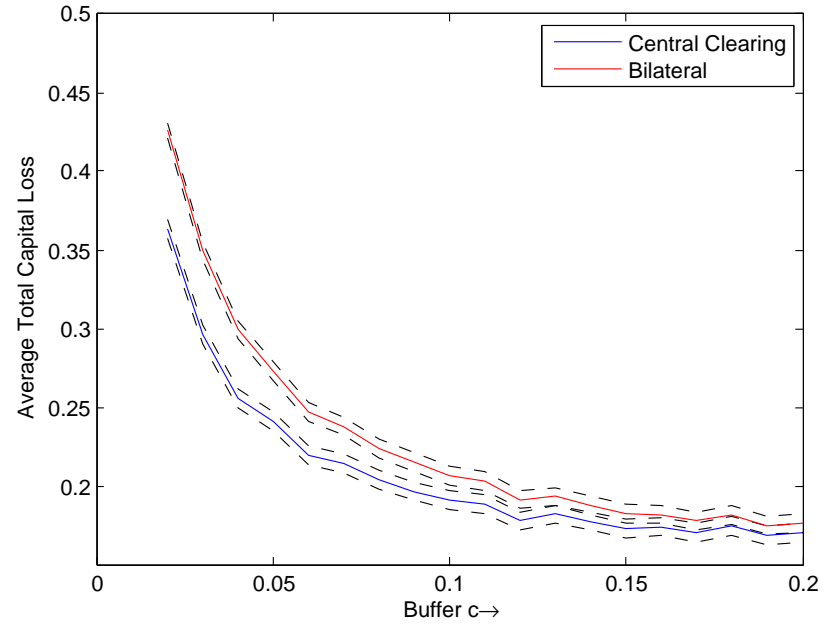

(b) Average Total Capital Loss

Finally, figure 6.10 shows the results of the probability of failure of the CCP for both settings considered above. Figure 6.10(a) shows that a higher capital buffer leads not only to a more stable system as we have seen earlier for the random network, but also leads to very remote probabilities that the CCP might fail as a result of a default of a GCM. As mentioned in the previous section, GCMs are less likely to default as a result of contagion because of the higher capital buffer. Therefore, simultaneously absorbing shocks they may face and mitigating any 
residual losses the CCP might have to cover in later default rounds. On the other hand, figure 6.10(b) shows the exact opposite result. The higher the capital buffer GCMs hold the higher the probability the CCP might not be able to perform on its obligations. The reason is similar to the one given above. A higher capital buffer means that large GCMs will only default when losses are extremely high. This hold especially for large GCMs. To illustrate this, we take the default probability of the CCP for a capital buffer of $15 \%$. We find in figure 6.10 (a) that the conditional probability that a CCP might fail given a random default is almost equal to zero. For the same point, we find in figure 6.10(b) that the conditional probability that a CCP might fail when a large GCM defaults equals more than $25 \%$. Given the fact that this scenario is contained in the simulation experiment results shown in figure 6.10(a), means that the probability of this happening is almost zero, but if this scenario occurs, in such case it might have an extreme negative impact on the CCP and the financial system as a whole in term of contagion of defaults. However, it should be noted that the contagion losses to the system as shown in figure 6.9(b) are still lower when OTC derivatives are centrally cleared with a CCP.

\section{Figure 6.10}

Probability of CCP Failure

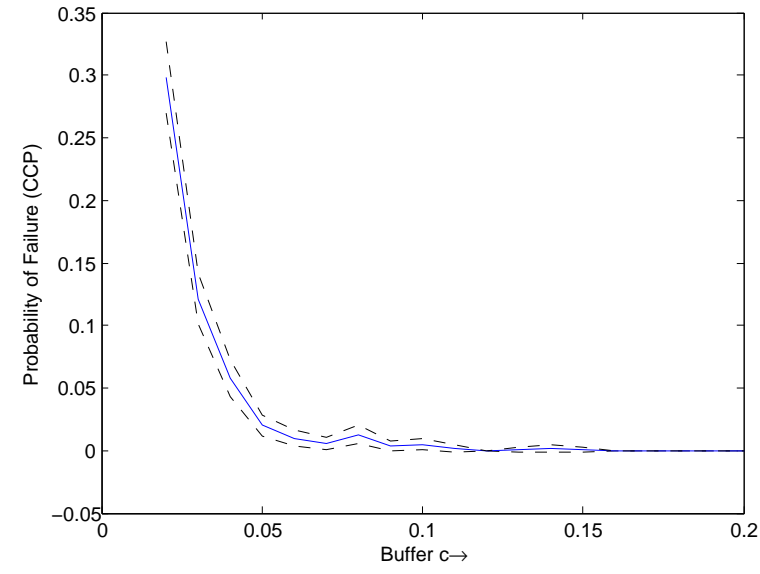

(a) CCP failure as a result from cascades from the failure of an arbitrary GCM

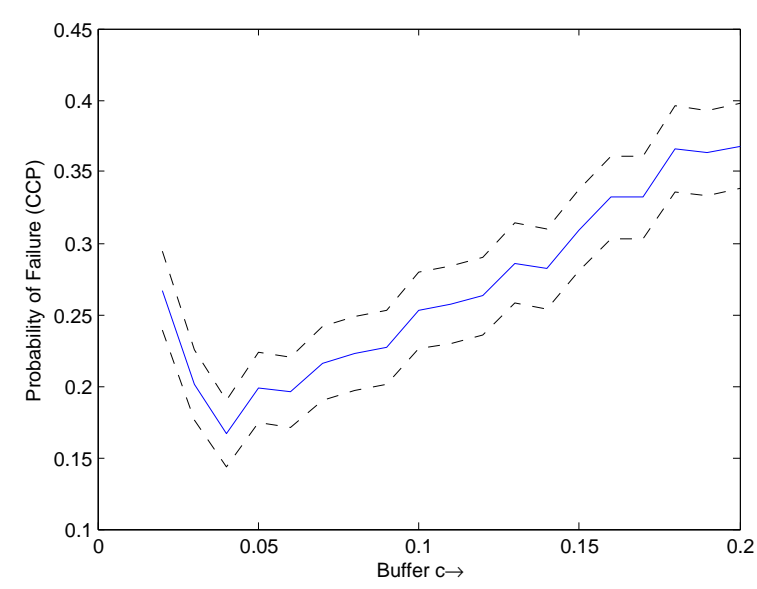

(b) CCP failure as a result from cascades from failure of an arbitrary large GCM

\subsection{Core-Periphery Tiered Structured Networks}

\subsubsection{Concentration and contagion}

In the previous section we conducted simulation experiments to assess the impact of central clearing of OTC derivatives on GCMs and the financial system in general. We specifically considered random and tiered random networks. The core-periphery structured networks reflect the topology of the financial system more realistically, which will be consider in the following experiments.

Figure 6.11(a) and figure 6.11(b) show the average default rates in a system where OTC derivatives are centrally cleared and bilateral cleared, respectively. We plot the average default 
rates for various shock sizes to see how the cascading failures compare in both systems. Unlike the results for the random networks, it appears that central clearing has no benefit to the financial system stability. Figure 6.11(b) shows that the average default rates are lower regardless of the number of counterparties in the system. In contrast, we find a linear increase of the average default rates in $n$ in figure 6.11(a). For more extreme shocks, we find no difference in default rates in both systems.

Figure 6.11

Default rates

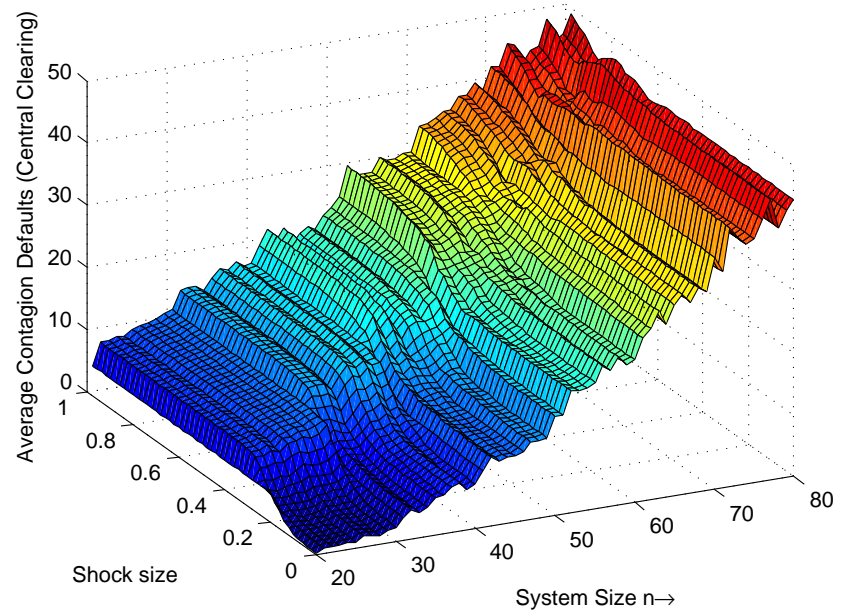

(a) Central Clearing

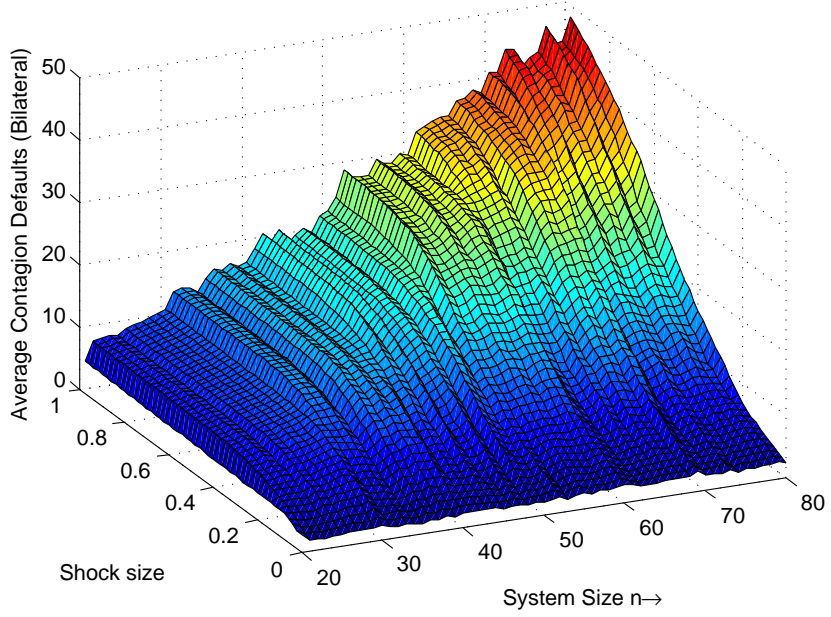

(b) Bilateral

The capital losses are given in figure 6.12(a) and 6.12(b), for a system with CCP and one without, respectively. Central clearing of OTC derivative appears to have some benefit when the losses are considered instead of the default rates. The simulation experiment shows that for very large shocks we obtain similar results in both systems. CCPs however appear to keep these losses limited over a considerable range in our simulation experiment, where we see a linear increase in losses when bilateral trades are considered. It is quite clear that the results in this setting where very large GCMs in the core and small GCMs in the periphery lead to a decrease in benefits of central clearing. The relative size of large GCMs translates to an increased complexity in managing contagion by the CCP in core-periphery structured networks. We further suspect that the linear increase in default rates is caused by large number of small GCMs defaulting as the result of one large GCM. Regarding bilateral clearing, defaulting counterparties in the core will most likely lead to a considerable spread of losses to other counterparties in the core because of their high interconnectivity. These parties have bigger capital buffers and hence are more capable of withstanding larger shock. This may explain the lower default rates in figure 6.12(b). Smaller GCMs get affected much faster when IRSs are centrally cleared.

The probability of failure of the CCP is given in figure 6.13. Again, unlike the results we obtained for the random network, we find a higher probability of failure of the CCP as $n$ grows. This leads us to conclude that large GCMs in the core can lead to considerable losses despite centrally clearing of OTC derivatives. Their high market capitalization decreases netting benefits and reduces diversification effects considerably. 
Figure 6.12

Average Total Capital Loss

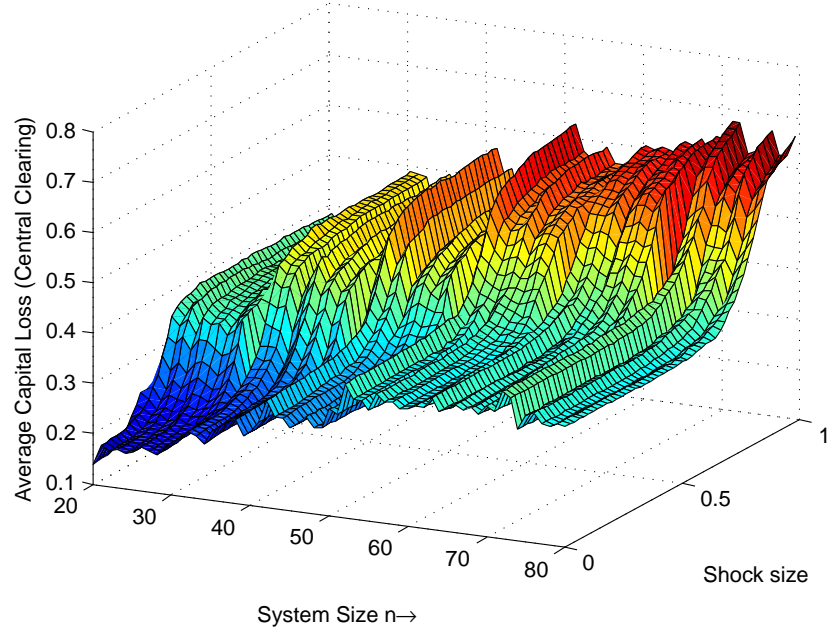

(a) Central Clearing

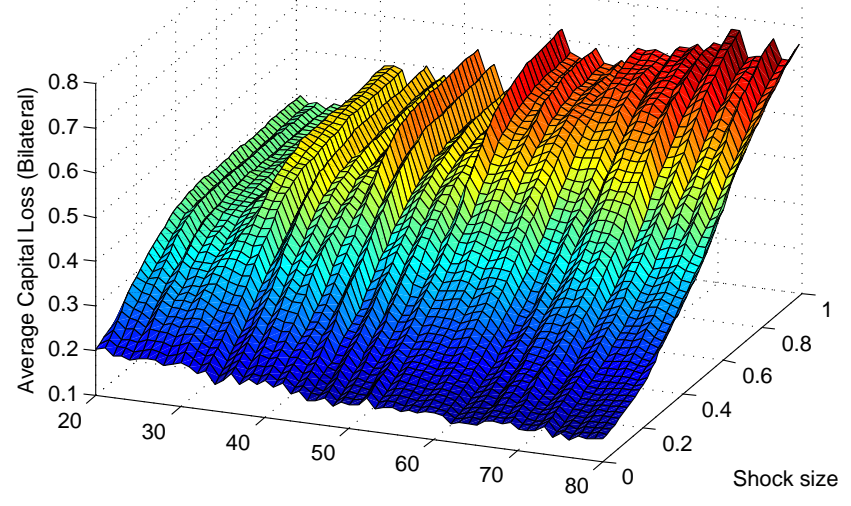

System Size $n \rightarrow$

(b) Bilateral

Figure 6.13

Probability of CCP Failure

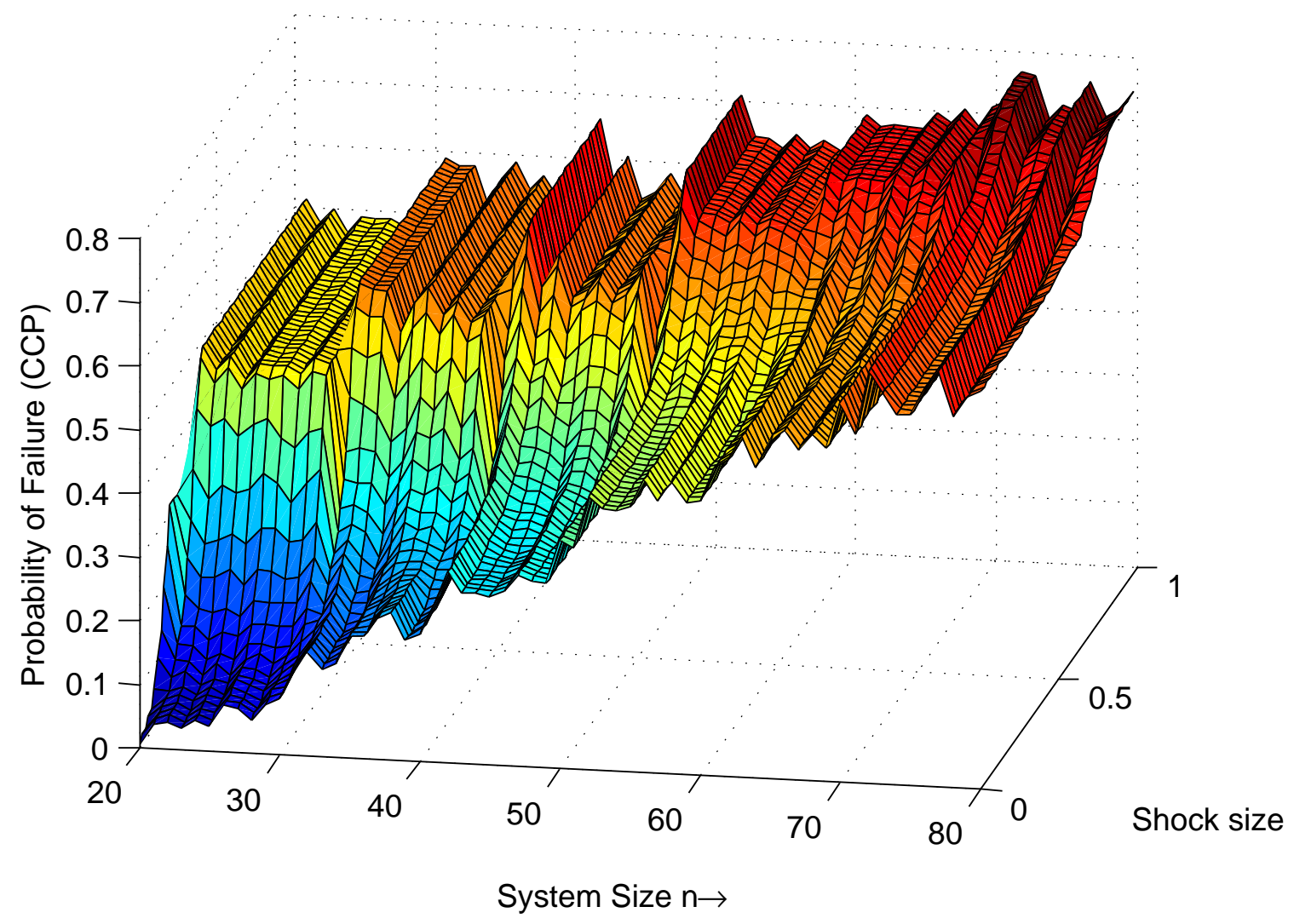

Considering the default rates and contagion losses from the failure of a large GCM, we obtain the results shown in figure 6.14 and figure 6.15. The results look similar to the ones obtained 
in the previous section for the random tiered networks. Central clearing of OTC derivatives does not lead to lower default rates but does lead to limited contagion losses to the financial system as a whole. Figure 6.15(a) shows that the benefit of central clearing is evident when more GCMs participate in the clearing system. Central clearing of OTC derivatives reduces the default rate quite significantly for $n>80$, thus leading to a noticeable more stable system in spite of the fact that more extreme shocks are introduced. Remarkably, the CCP is still able to cover the residual losses. This jump not only confirms the non-linearity between the average default rates and contagion losses, but also shows how complex the relationship between default rate and concentration can be even when only one parameter is varied.

Figure 6.14

Default rates

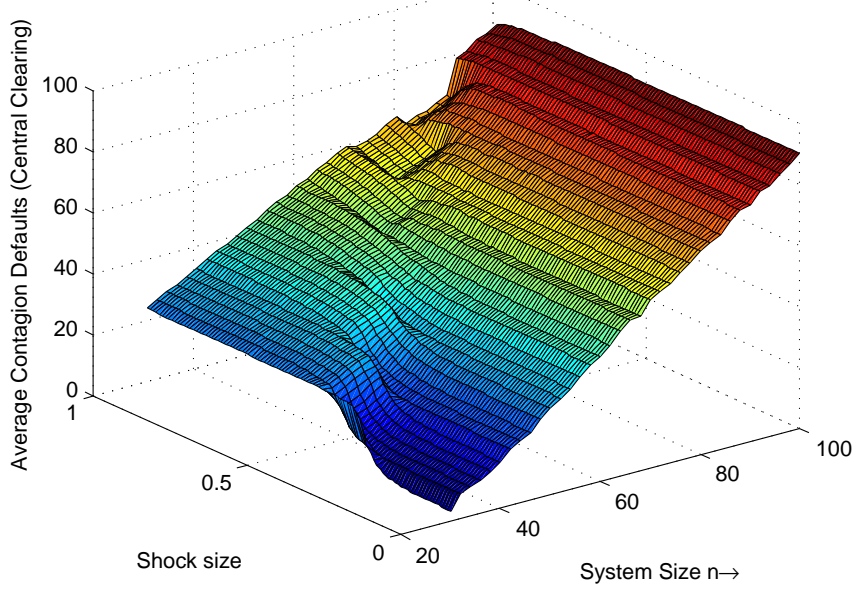

(a) Central Clearing

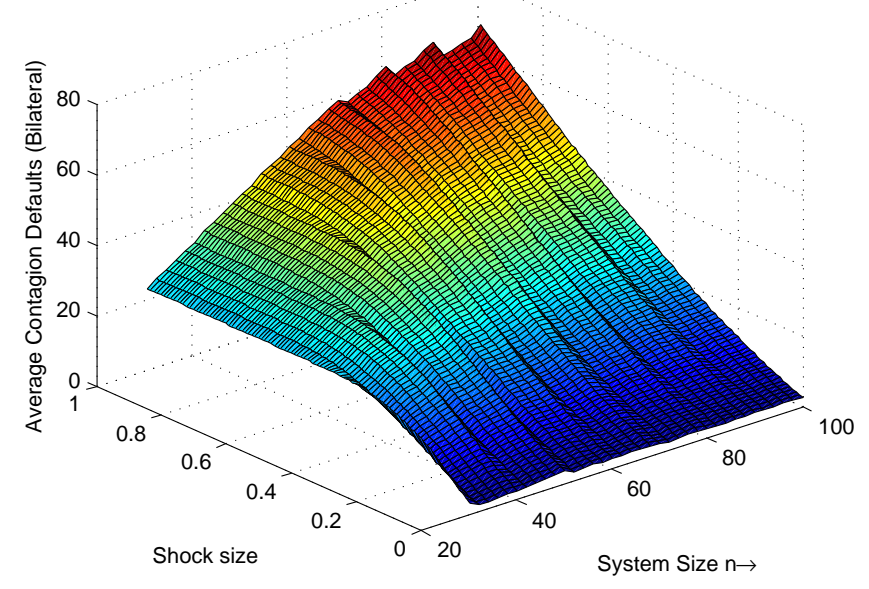

(b) Default rates (Bilateral)

Figure 6.15

Average Total Capital Loss

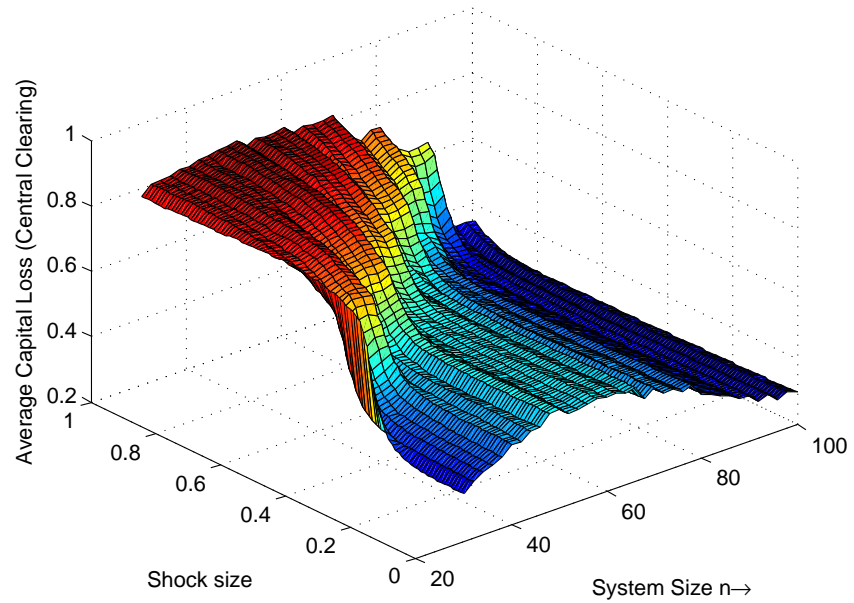

(a) Central Clearing

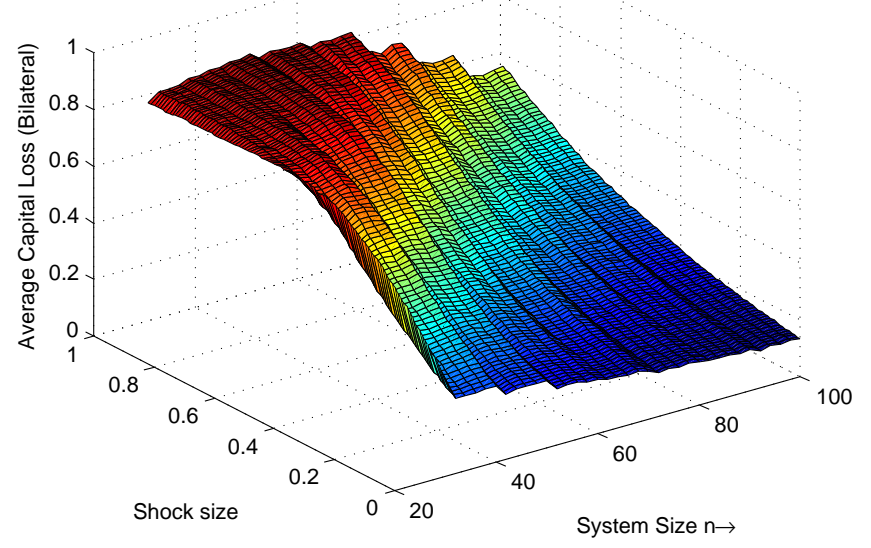

(b) Bilateral 
The probabilities of failure of the CCP as a result of contagion from the default of a core GCM are shown in figure 6.16. The results are comparable to the experiment result shown in figure 6.13, where contagion from an arbitrary default is considered. The most notable result we obtain from this experiment is that if the shock is big enough, the CCP will most surly get into trouble, unlike the case shown in figure 6.13, where we obtained high probabilities of failure but these probabilities do not converge to 1 . These results show that too big too fail GCMs in the core still negatively affect the financial system even when OTC derivatives are centrally cleared if the losses are big enough.

Figure 6.16

Probability of CCP Failure

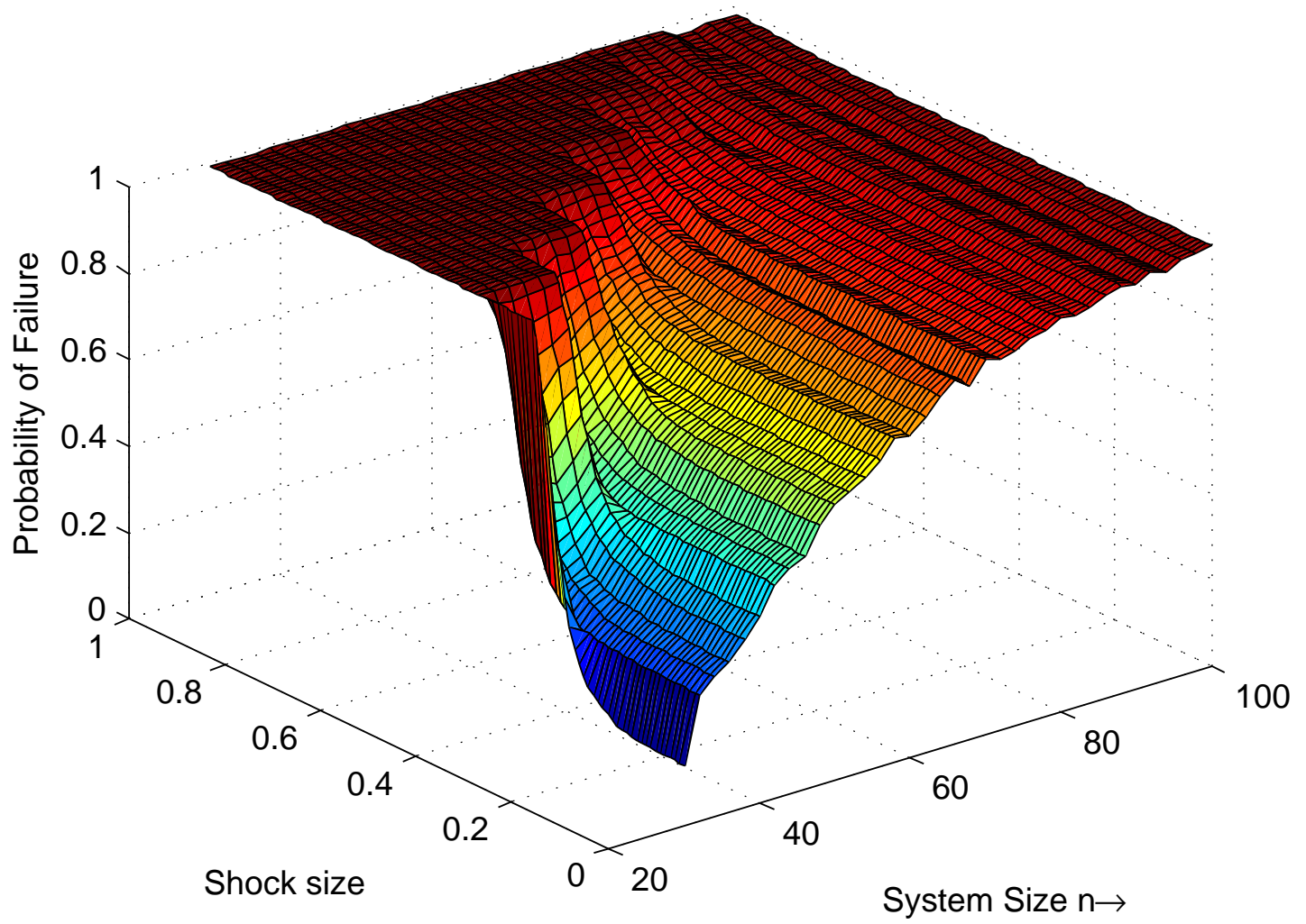

\subsubsection{Capitalization and contagion}

Lastly, simulation experiments are carried out on the impact of capitalization on the contagion of defaults and losses. Figure 6.17 and figure 6.18 show the average contagion default rates and average capital losses, respectively. The results appear to be in line with our intuition and with the results obtained for the random networks. A higher capital buffer leads to a much more stable system and limits both the default rates as the losses considerably. $c=15 \%$, which is significantly high compared to more practical rang of $c<0.08$, is sufficient to dampen the contagion from defaults. Central clearing of OTC derivatives appears to have a slight benefit over a system where IRSs are bilaterally traded. Figure 6.17(a) shows that the default rates drop slightly faster when IRSs are centrally cleared combined with a higher capital buffer held 
by GCMs.

Figure 6.17

Default rates

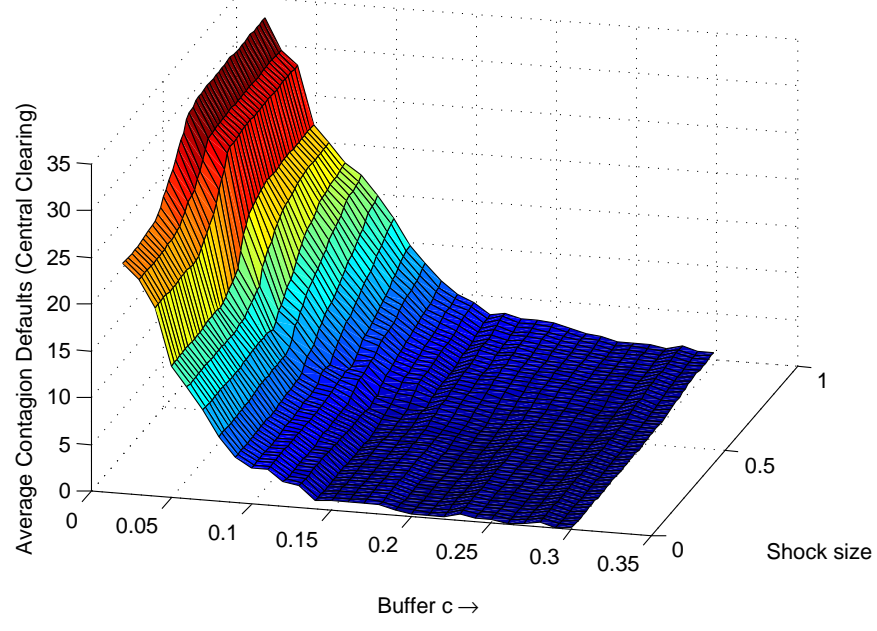

(a) Central Clearing

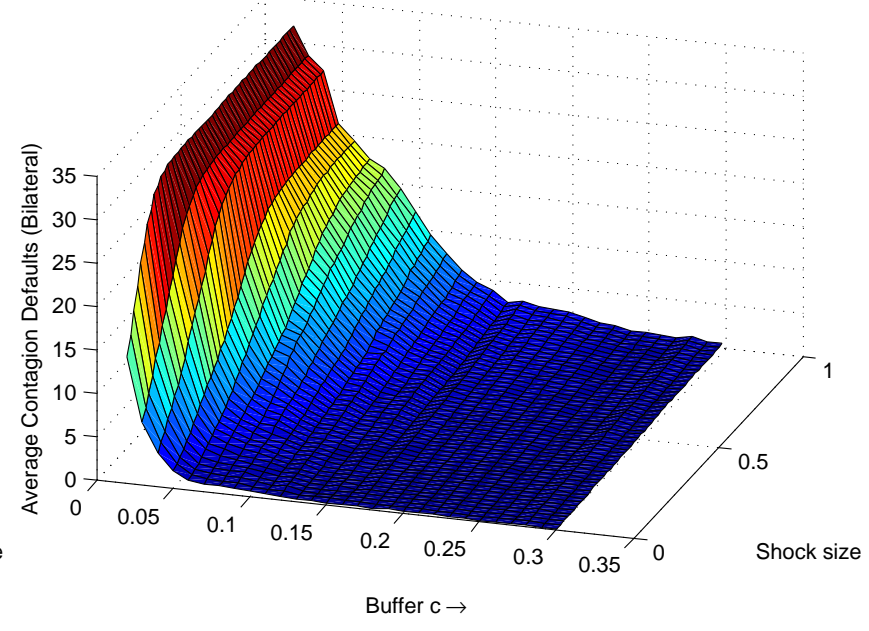

(b) Bilateral

Figure 6.18

Average Total Capital Loss

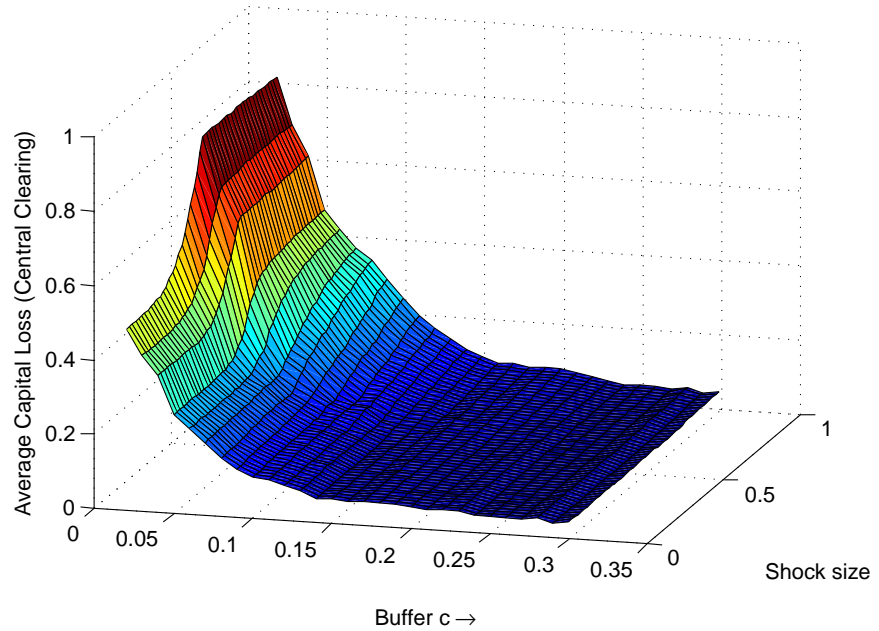

(a) Central Clearing

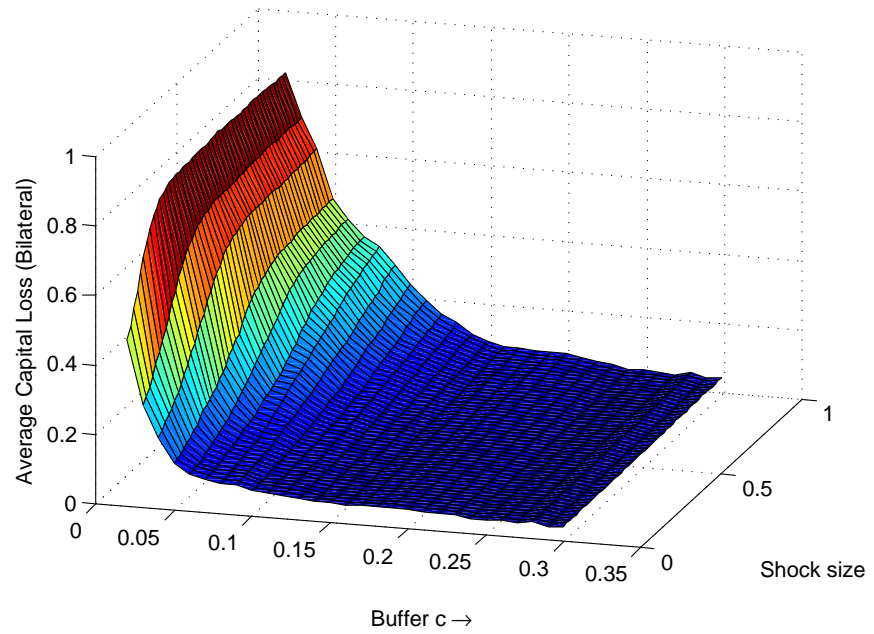

(b) Bilateral

Figure 6.20 shows the probability of failure given the aggregate capitalization. These results are in conformity with the statistics for the random tiered network. The CCP benefits from a higher capitalization of GCMs, since any residual losses are much better covered by GCMs. Contagion defaults and losses are thus much lower. This relieves both GCMs and the CCP from residual losses in later default rounds.

The analyses is completed by considering contagion from the default of a core GCM. The results are based on the same simulation experiment as before except that only fundamental 
Figure 6.19

Probability of CCP Failure

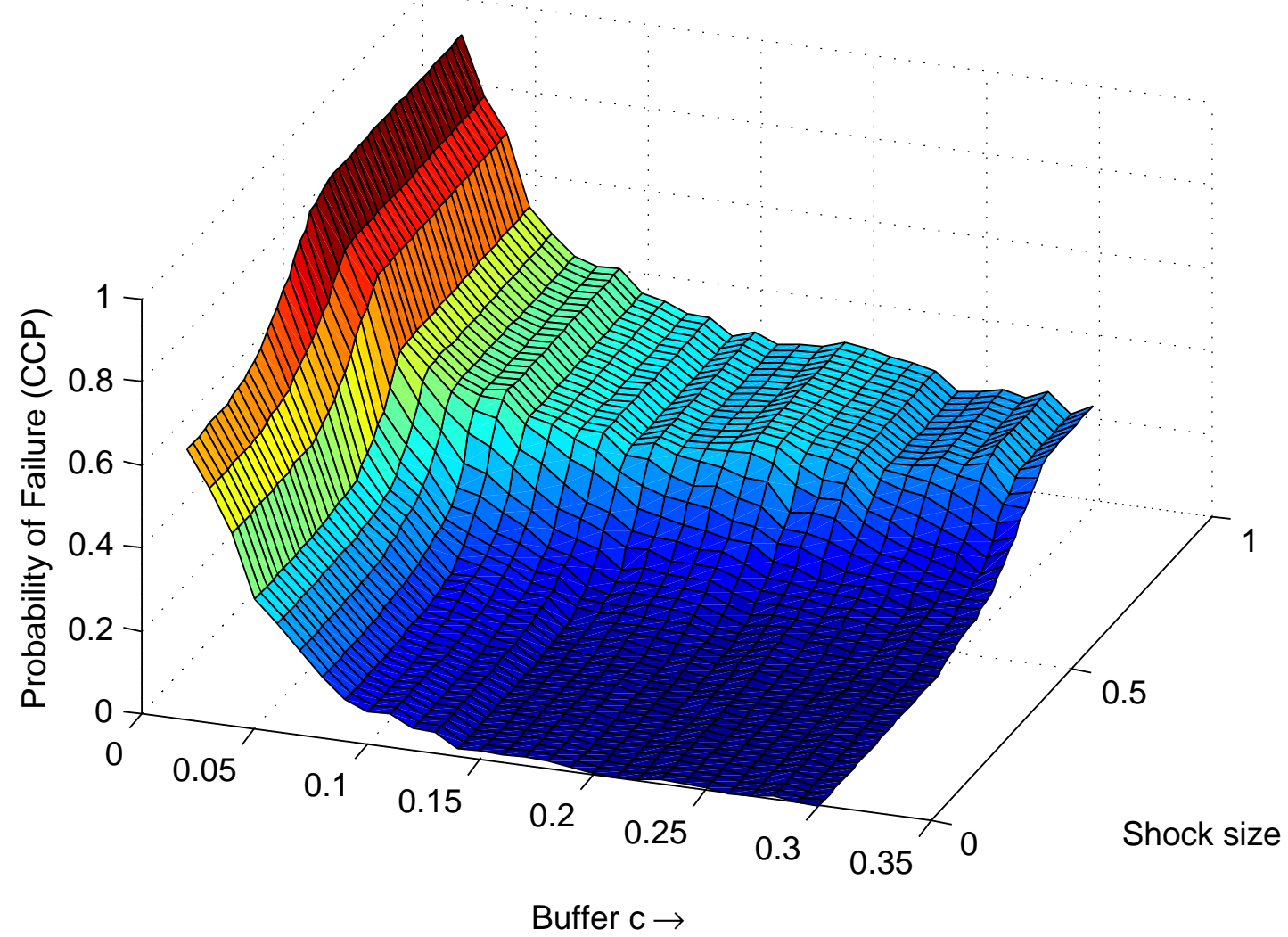

defaults of core GCMs are taken into consideration. The results are given in figure 6.20 and figure 6.21. A higher capitalization of GCMs appears to stabilize the system considerably as we have seen before. Recall that contagion in a random tiered network was prominent regardless of the aggregate capitalization, as shown in figure 6.9. In contrast, we find that contagion from the default of a core GCM does not appear to have such an effect on the system either with or without a CCP when a core periphery tiered structured network is considered. Our analysis suggests that the specific network structure leads to such different effects of contagion. Large counterparties in random tiered networks have a high linkage to other large counterparties as to small counterparties alike. This is not the case for core periphery structured networks where counterparties are strongly connected to other counterparties in the core but only have a few connections to counterparties in the periphery. On average, contagion spreads more easily to many small counterparties when a large counterparty defaults in random networks. In a core periphery structured network, the default of a core counterparty will lead to a large spill over of losses to other counterparties in the core. Only if the shock is big enough to lead to more defaults in the core, will small counterparties eventually be affected on a large scale. It could be said that a considerable amount of counterparties in the periphery is shielded from losses by their counterparty in the core unlike the random tiered network where this is not the case. This explains why the contagion default rates and losses are much lower in a core-periphery structured network with bilateral clearing. A higher capitalization of GCMs (counterparties) means a higher capitalization of core GCMs (counterparties) and hence a stable financial system 
with or without central clearing.

Figure 6.20

Default rates

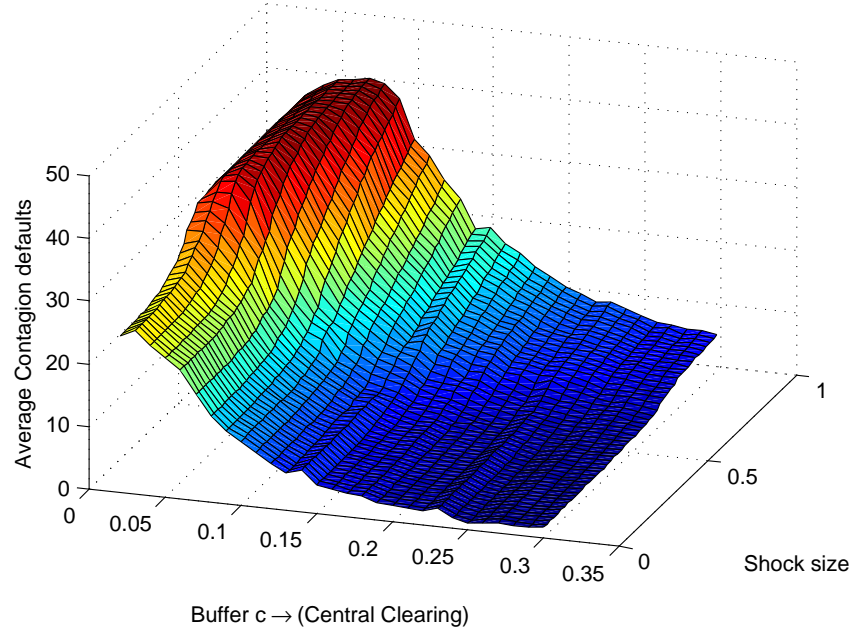

(a) Central Clearing

Figure 6.21

Average Total Capital Loss

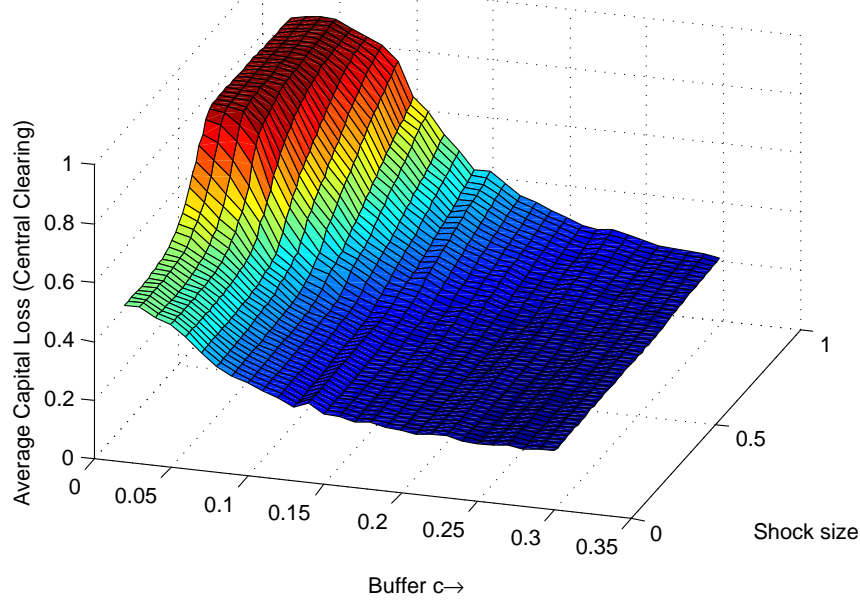

(a) Central Clearing

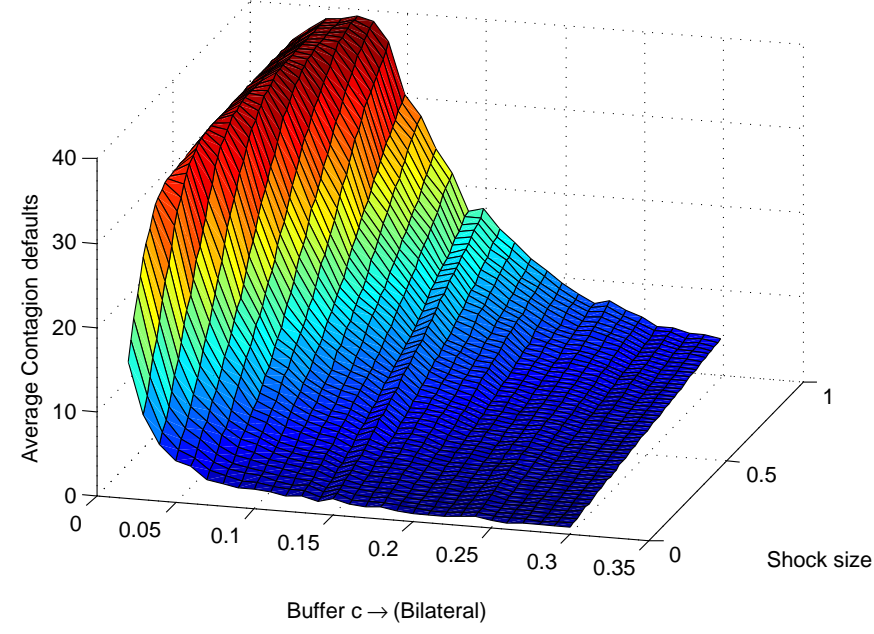

(b) Bilateral

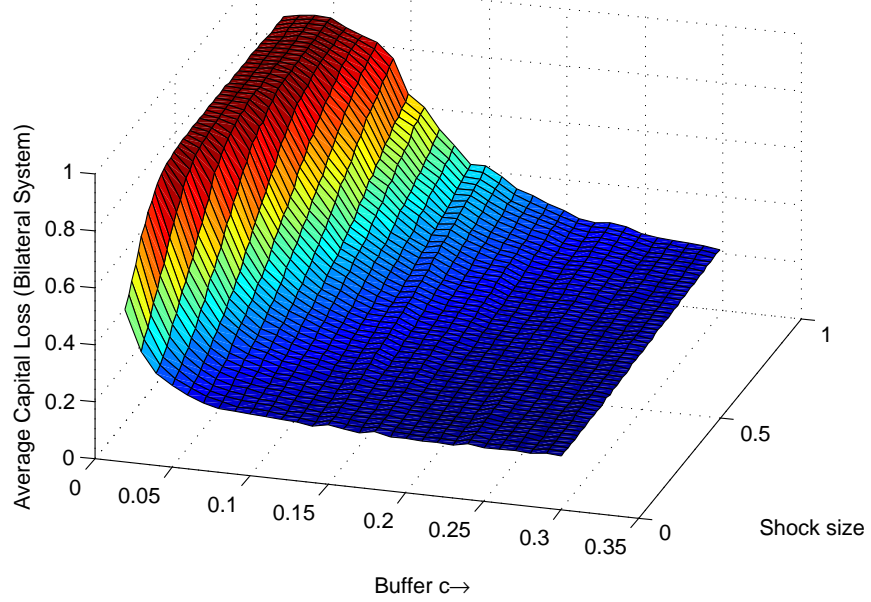

(b) Bilateral

Lastly, the failure rates of the $\mathrm{CCP}$ given the aggregate capitalization of the system are given in figure 6.22. CCPs benefit considerably from higher capital buffers held by GCMs. We find that the failure rates stabilize for $c>15 \%$ for different shock sizes. However, a sharp jump in failure rates for 'moderate' to large shock sizes is in contrast to the results in figure 6.19. Core GCMs with a large buffer can withstand large shocks, but when a default happens, the CCP appears to be unable to cover for these losses. This however does not lead to a complete system break down as long as other core GCMs have a buffer that is enough the absorb any contagion losses as we have shown in figure 6.20(a) and 6.21(a). 
Figure 6.22

Probability of CCP Failure

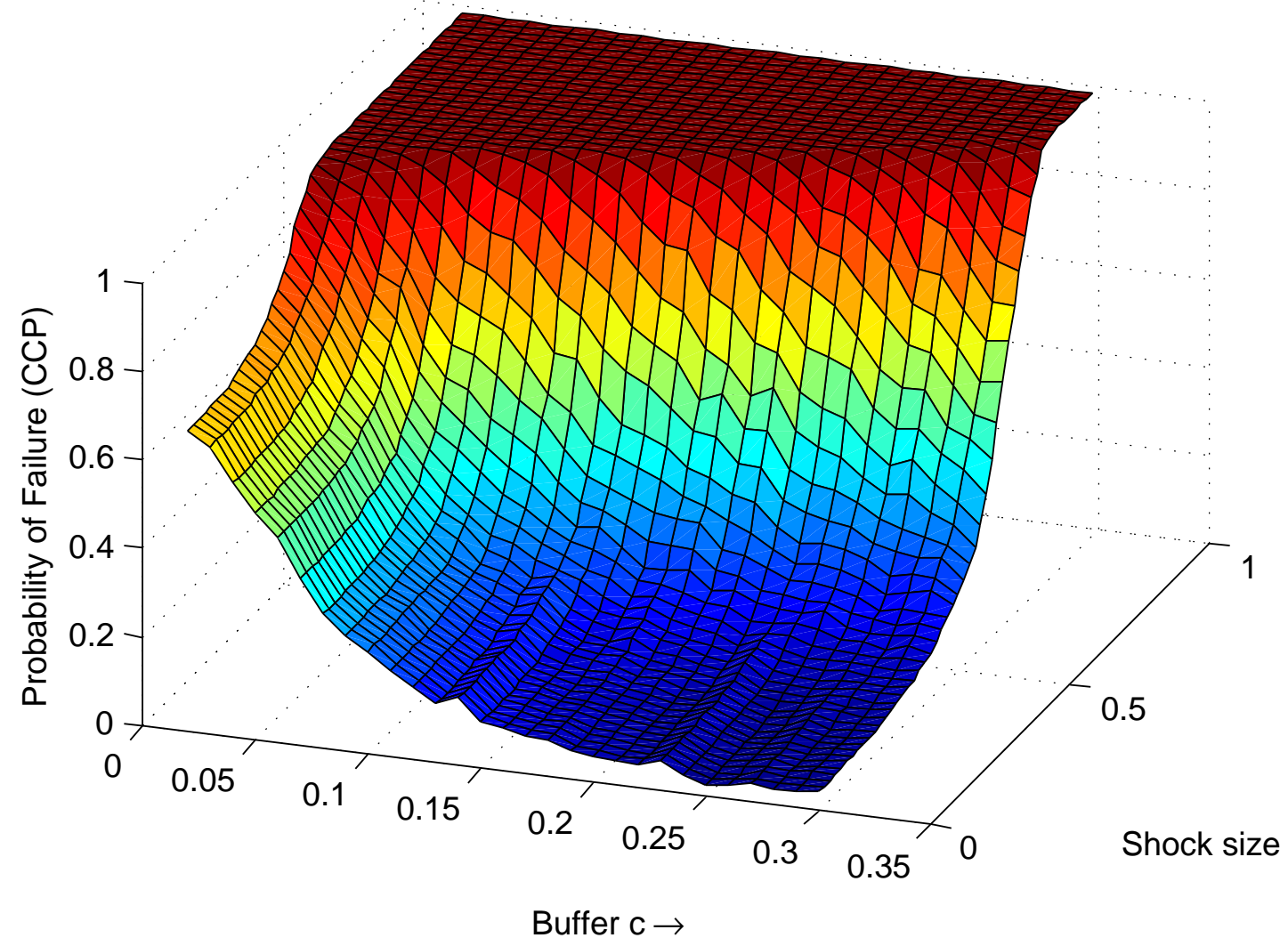




\section{Conclusions}

This paper examines how central clearing of OTC derivatives impactss systemic risk and contagion in financial systems. We consider a set of network topologies, which are typically used to analyse financial systems. We distinguish between Erdös-Rényi networks and small-world networks. Our aim was to keep the model as simple as possible on the one hand, but also as close to reality as possible on the other. For this purpose, we applied simplified versions of many clearing mechanisms available to CCPs.

We analysed how the system risk and contagion behave when different network configuration are considered and how these depend on concentration and capitalization of the market participants. Our analysis is comparative in nature, as our main purpose was to assess in how far CCPs mitigate system risk (or perhaps aggravate losses), compared to the current situation of bilateral OTC derivatives markets. Our approach of considering interest rate swaps and perturbing the yield curve enabled us to simulate both fundamental defaults (which are the cause of contagion), as well as exposures within the system in one single step. Contagion defaults and losses were subsequently determined in a recursive way.

We have shown that the effect of the central clearing on the financial system is complex and highly non-linear. The first part of the analysis focused on random networks, where all parties are similarly connected and are of similar size. The CCP successfully mitigates system risk in such a setting, in terms of both contagion default rates and capital losses. This setting, however, does not represent financial networks in a realistic way: financial institutions are generally of different sizes and different degree of connectedness.

A more realistic setting is characterized by tiering, i.e., introducing a small number of large and highly connected financial institutions. Overall, it seems that also in this setting the CCP is capable of reducing counterparty credit risk. However, when a fundamental default affects a large financial institution, we find that, in presence of CCP, contagion default rates are significantly higher than for a bilateral market. On the other hand, total capital losses are still lower in presence of CCP: this follows from the fact that higher default rates are caused by many defaulting smaller clearing members, which are disproportionately sacrificed in the event of market stress.

Finally, a core-periphery structured network is implemented, representing the most realistic setting. In this case, the size and connectedness of financial institutions differ even more, so we find that the CCP has much more trouble reducing counterparty risk. Small counterparties in a bilateral OTC market are not directly affected by contagion when they are shielded away from the rest of the market. This follows from the fact that these parties are generally only connected to one counterparty in the core and none in the periphery. In contrast, central clearing translates to a much wider spread of contagion to small institutions in the periphery, which subsequently instigates a high default rate when losses from a large clearing member are too big for the CCP to handle. Capital losses still appear to be somehow limited by the CCP, even when this extreme case is considered. To mitigate this effect, a "social cost" of default must be 
introduced into a financial system.

The impact of central clearing of OTC derivatives on system risk is complex and a network structure heavily affects how defaults propagate through the system. In how far the CCP successfully mitigates system risk depends on which financial institutions first experience fundamental defaults. If a large financial institution defaults due to some fundamental reason, such as an adverse shock to the interest rates, small financial institution suffer much more from contagion - the effect which is further exacerbated by the central clearing. So it appears that for the sake of financial system stability it is more useful to concentrate regulatory efforts on core/large financial institutions. 


\section{References}

Barbasi A.-L. \& R. Albert (1999), Emergence of Scaling in Random Networks, Science 286, pp. 509-512.

Blundell-Wignall A. \& P. Atkinson (2011), Global SIFIs, Derivatives and Financial Stability, OECD Journal: Financial Market Trends, Issue 1, pp. 1-34.

Cont R., A. Moussa \& E.B. Santos (2010), Network structure and systemic risk in banking systems, Available at SSRN: http://ssrn.com/abstract=1733528

Craig B. \& G. von Peter (2010), Interbank Tiering and Money Center Banks, BIS 322, Basel.

Degryse H. \& G. Nguyen (2004), Interbank exposure: an empirical examination of system risk in Belgian banking system, CentER Discussion Paper No. 04.

Elsinger H., A. Lehar \& M. Summer (2006), Using Market Information for Banking System, International Journal of Central Banking 2, Issue 1, pp. 137-165.

Erdős P. \& A. Rényi (1959), On Random Graphs, Publicationes Mathematicae 6, pp. 290-297.

Erdős P. \& A. Rényi (1960), On the Evolution of Random Graphs, Publ. Math. Inst. Hungar. Acad. Sci 5, pp. 17-61.

Franklin A. \& G. Douglas (2000), Financial Contagion, Journal of Political Economy 108, Issue 1, pp. 1-33.

Furfine C.H. (2003), Interbank Exposure: Quantifying the Risk of Contagion, Journal of Money, Credit and Banking 35, Issue 1, pp. 111-28.

Fricke D. \& T. Lux (2012), Core-Periphery Structure in the Overnight Money Market: Evidence from the e-Mid Trading Platform, Kiel Institute for the World Economy, Working Paper No. 1759 .

Gai P. \& S. Kapadia (2010), Contagion in Financial Networks, Proc. R. Soc. 406, pp. 2401-2423.

Gai P., A. Haldane \& S. Kapadia (2011), Complexity, Concentration and Contagion, Journal 
of Monetary Economics58, Issue 5, pp. 453-470.

van Lelyveld I. \& F. Liedorp (2006), Interbank Contagion in the Banking Sector: A Sensitivity Analyses, International Journal of Central Banking 2, Issue 2, pp. 99-133

Newman M.E.J., S.H. Strogatz \& D.J. Watts (2001), Random graphs with arbitrary degree distributions and their applications, Physical Review E 64, p. 026118

Markose S. (2012), Systemic Risk from Global Financial Derivatives: A Network Analysis of Contagion and Its Mitigation with Super-Spreader Tax, IMF Working Paper No. 12/282.

Markose S., S. Giansante \& A.R. 'Shaghaghi (2012), Too Interconnected to Fail' Financial Network of IS CDS Market: Topological Fragility and Systemic Risk, Journal of Economic Behavior $\&$ Organization 83, Issue 3, pp. 627-646.

Milgram S. (1967), The Small-World Problem, Psychology Today 2, pp. 61-67.

Nier E., J. Yang, T. Yorulmazer \& A. Alentorn (2007), Network Models and Financial Stability, Journal of Economics Dynamics and Control 31, Issue 6, pp. 2033-60.

Pirrong C. (2011), The Economics of Central Clearing: Theory and Practice, ISDA Discussion Papers Series No. 1.

Pykhtin M. \& S. Zhu (2007), A Guide to Modelling Counterparty Credit Risk, GARP Risk Review, Issue 37, pp. 16-22.

Ripatti K. (2004), Central Counterparty Clearing: Constructing Framework for Evaluation of Risks and Benefits, Bank of Finland Research Discussion Paper No. 01/04.

Watts D.J. (1999), Networks, Dynamics and the Small-World Phenomenon, American Journal of Sociology 105, Issue 2, pp. 493-527.

Watts D.J. (2002), A Simple Model of Global Cascades on Random Networks, Proceedings of the National Academy of Sciences of the United States of America 99, No. 9, pp. $5766-5771$.

Watts D.J., S.H. Strogatz (1998), Collective Dynamics of 'Small-World' Networks, Nature 393, pp. $440-442$.

Upper C. (2007), Using Counterfactual Simulation to Assess the Danger of Contagion in Interbank Markets, BIS Working Papers No. 234, Basel.

Upper C. (2011), Simulation Methods to Assess the Danger of Contagion in Interbank Markets, Journal of Fiancial Stability 7, pp. 111-125. 
Upper C. \& A. Andreas (2004), Estimating Bilateral Exposures in the German Interbank Market: is there a Danger of Contagion?, Deutsche Bundesbank, European Economic Review 48, pp. 827-849. 